\title{
Equilibrium of an arbitrary bunch train in presence of a passive harmonic cavity: Solution through coupled Haïssinski equations
}

\author{
Robert Warnock $\odot^{*}$ \\ SLAC National Accelerator Laboratory, Stanford University, Menlo Park, California 94025, USA, \\ and Department of Mathematics and Statistics, University of New Mexico, \\ Albuquerque, New Mexico 87131, USA \\ Marco Venturini ${ }^{\dagger}$ \\ Lawrence Berkeley National Laboratory, University of California, Berkeley, California 94720, USA
}

(Received 22 March 2020; accepted 12 May 2020; published 24 June 2020)

\begin{abstract}
We study the effect of a passive harmonic cavity, introduced to cause bunch lengthening, in an electron storage ring. We derive a formula for the induced voltage from such a cavity with high $Q$, excited by a sequence of bunches, allowing for arbitrary gaps in the sequence and arbitrary currents. Except for a minor term that can be determined iteratively, the voltage is given in terms of a single mode of the Fourier transforms of the bunch forms, namely, the mode at the resonant frequency of the cavity. Supposing that the only wakefield is from the harmonic cavity, we derive a system of coupled Haïssinski equations which determine the bunch positions and profiles in the equilibrium state. The number of unknowns in the system is only twice the number of bunches, and it can be solved quickly by a Newton iteration, starting with a guess determined by path following from a solution at a weak current. We explore the effect of the fill pattern on the bunch lengthening and also the dependence on the shunt impedance and detuning of the cavity away from the third harmonic of the main accelerating cavity. We consider two measures to reduce the effects of gaps: (i) distribution of the gaps around the ring to the greatest extent allowed and (ii) "guard bunches" with higher charges adjacent to the gaps, compensating for the charge missing in gaps. Results for parameters of the forthcoming Advanced Light Source upgrade are presented.
\end{abstract}

DOI: 10.1103/PhysRevAccelBeams.23.064403

\section{INTRODUCTION}

In electron storage rings, the phenomenon of Touschek scattering often limits the lifetime of a stored beam [1]. This is the aspect of intrabeam scattering in which small transverse momenta are transformed through Coulomb scattering and a Lorentz boost into one large and one small longitudinal momentum in the lab frame, sending both particles outside the momentum aperture of the ring. The effect may be counteracted by reducing the charge density in the beam. One way to do that is to increase the bunch size in the longitudinal direction. This can be done by adding a cavity with resonant frequency close to a low harmonic of the main rf frequency, say, the third or fourth harmonic. This is often called a higher-harmonic cavity (HHC).

\footnotetext{
warnock@slac.stanford.edu

mventurini@lbl.gov
}

Published by the American Physical Society under the terms of the Creative Commons Attribution 4.0 International license. Further distribution of this work must maintain attribution to the author(s) and the published article's title, journal citation, and DOI.
The quadratic potential well of a usual rf system can be turned into a quartic potential well, by arranging the $\mathrm{HHC}$ so as to zero the second and third derivatives of the effective well $[2,3]$. This condition, often referred to as "ideal", results in a flattop equilibrium bunch profile with a substantial bunch length increase, say, by a factor of 4 or more in cases of interest, and an increase of the Touschek lifetime by a comparable factor. However, the flattop is not necessarily the best configuration, since a further lifetime improvement can be achieved by "overstretching", which causes the appearance of two peaks in the bunch profile. This must not be carried too far, however, since eventually the average lifetime will degrade rather than improve with overstretching.

Higher-harmonic cavities for bunch lengthening have long been in use at several third-generation light sources, including National Swedish Synchrotron Radiation Facility (MAX-II, now closed) [4], Advanced Light Source (ALS) [5], Swiss Light Source (SLS) [6], Sincrotrone Trieste (ELLETRA) [7], Berlin electron-storage ring (BESSY) [8] and in the Double Annular $\Phi$ Factory (DAFNE $e^{+}-e^{-}$ collider) [9]. There is now renewed interest driven by the trend toward ultralow emittance, which is making the new generation of light sources increasingly sensitive to scattering effects. A possible installation at National 
Synchrotron Light Source (NSLS-II) is being evaluated [10-12], and the fourth-generation light source MAX-IV has a system already commissioned [13]. Forthcoming fourth-generation machines, including Advanced Light Source upgrade (ALS-U) [14], Advanced Photon Source upgrade (APS-U) [15], Brazilian Synchrotron Light Source (SIRIUS) [16], Upgrade of synchrotron light source at DESY (Deutsches Elektronen- Synchrotron) (PETRA-IV) [17], Swiss Light Source upgrade (SLS-2) [18], European Synchrotron Radiation Facility upgrade (ESRF-EBS) [19], Diamond Light Source upgrade (Diamond-II) [20], and Hefei Light Source upgrade (HLS-II) [21] all have harmonic cavities as essential components.

The harmonic cavity may be passive or actively excited, but a natural first step is to consider the less expensive passive option. Our discussion is for the passive case, but our methods could be adapted to the active system. In the passive case, the field induced in the cavity by a bunch train depends strongly on the fill pattern. If the beam has a uniform fill pattern, e.g., all rf buckets are filled or all the bunches are separated by a fixed number of empty rf buckets, there exists a beam equilibrium with all bunches having the same profile (possibly of the flattop form if the ideal HHC settings are met). However, if there are significant gaps between bunch trains (or a long gap following a single-train beam), the quality of the beam equilibrium can be compromised. Instead of uniform charge distributions along the train, one then sees a variation of the bunch form and centroid position along the train. This may cause severe limitations to the effectiveness of the HHC system, either because of the resulting uneven lifetime or/and because of interference with the functioning of the machine feedback systems used for beam stabilization, and may prevent the attainment of the desired bunch lengthening.

There are several reasons for the presence of gaps in the bunch train. Historically, gaps have been needed for ion clearing. Another demand arises from the requirements of synchrotron light users, who may need different fill patterns for different types of experiments. Experiments needing precise timing of $\mathrm{x}$-ray pulses generally require more gaps than those asking for high brilliance. In the ALS-U, gaps are needed for on-axis injection from the accumulator ring [22].

In this paper, we present a robust and efficient method to evaluate the beam equilibrium for arbitrary HHC settings and beam-fill patterns. Our approach, entailing the numerical solution of a system of nonlinear algebraic equations, extends the method introduced in Ref. [23] for the determination of single-bunch Haïssinski equilibria in the case of short-range wakefields. It is much faster than macroparticle-based methods and, we believe, an improvement on the method recently introduced by Olsson, Cullinan, and Andersson [24].

Our immediate objective is to study the effect of the fill pattern on the bunch densities in the equilibrium state. While this is a useful first step with rewarding practical implications, e.g., offering guidance on the choice of HHC design parameters, our final goal is to understand the threshold in current for an instability and the timedependent behavior beyond the threshold.

The widened potential well has some benefits: The reduced peak bunch current and increased longitudinal tune spread may lead to the damping of certain instabilities. However, other instabilities may be induced, either through the fundamental or higher-order modes of the HHC [25-28] or by possibly aggravating the effect of higherorder modes in the main cavity [29]. The method presented here is an essential ingredient toward the application of mode-analysis techniques to the study of beam stability when HHCs are present.

Besides reports on specific projects as cited above, there are several papers which discuss the issues that concern us in a more or less general way, through theory, simulations, and measurements. Byrd and Georgsson [3] and Hofmann and Myers [2] treated the situation without HHC beam loading (i.e., without the cavity wakefield), which is the starting point for the present work. Towne [30] studied stability of stretched bunches in the presence of a broad band impedance together with a high- $Q$ resonator, using Vlasov-Fokker-Planck simulations and measurements at the NSLS vacuum ultraviolet ring (VUV). Byrd, De Santis, Jacob, and Serriere [31] initiated the study of the impact of gaps in the bunch train. They used the term "transient beam loading", which several authors have adopted. (Since a transient effect is usually thought of as short-lived in time, not the case here, "inhomogeneous beam loading" might be a more descriptive term.) A direct antecedent of our work is the paper of Tavares, Andersson, Hansson, and Breunlin [32], who were concerned with self-consistency in the equilibrium bunch densities. The study of this topic was continued by Olsson, Cullinan, and Andersson [24], who developed an iterative scheme to find the equilibrium charge densities. Bassi and Tagger [12] investigated the option of a superconducting HHC, invoking self-consistent simulations and emphasizing the importance of beam loading in the main accelerating cavity for a full picture. Yamamoto, Takahashi, and Sakanaka [33] propose a special design of the HHC and a feed-forward system to compensate for gaps. Wüustefeld, Jankowiak, Knobloch, and Ries [34] and also Tian, Jiao, and Wang [35] proposed the production of simultaneous long and short bunches by using two harmonic cavities

The content of the paper is as follows.

Section II describes our choice of coordinates and the description of the bunch train. Section III and the Appendix A review the equations of motion.

Section IV states the primary formula for the voltage induced by the harmonic cavity, and then Sec. V notes that the induced voltage can be expressed in terms of an effective wake potential, which is represented by a compact formula that is the basis for further work. Section VI goes on to find an explicit formula for the induced voltage from 
an arbitrary bunch train, which is in terms of the Fourier transforms of the bunch forms at the resonant frequency of the harmonic cavity.

Section VII states the Vlasov-Fokker-Planck equation and shows how its steady state solution is given by the solution of coupled Haïssinski equations. Section VIII shows that the mean energy transfer in the equilibrium state is exactly equal to the energy loss per turn. Section IX calculates the integral of the induced voltage, to get the potential wells for the Haïssinski system.

Section X describes a Newton iteration for a solution of the Haïssinski system, while Sec. XI gives the associated Jacobian matrix, and Sec. XII shows how to follow the Newton solution as a function of the current.

Section XIII presents numerical results for the parameters of ALS-U and a comparison to a macroparticle simulation. Section XIV estimates Touschek lifetimes as a function of the cavity detuning.

Appendix B discusses the perturbation of the synchronous phase due to the harmonic cavity and reports that there is no necessity to base the coordinate system on the perturbed phase. Appendix $\mathrm{C}$ explains how our general formula for the induced voltage reduces to a known formula in the case where all bunches are identical.

\section{CHOICE OF VARIABLES AND DESCRIPTION OF BUNCH TRAIN}

Synchrotron motion in a storage ring can be described in terms of the longitudinal coordinate $z=\beta_{0} c t-s$, the distance to the reference particle. Here, $s$ measures position in the laboratory as arc length along a reference trajectory, and the reference particle has position $s_{0}=\beta_{0} c t$ at time $t$. Particles leading the reference particle have $z<0$. The opposite sign convention is often adopted, indeed in our own papers.

For a single bunch, $z$ is familiar as the "beam frame coordinate", which is suitable as a phase space coordinate for equations of motion and the Vlasov equation. In the case of many bunches, $z$ is a convenient global coordinate for the description of the total charge density, and merely by adding constants to $z$ we can construct local beam frame coordinates for all the bunches. Moreover, $z$ has the convenient property of being proportional to $s$ at fixed $t$ and proportional to $t$ at fixed $s$. Thus, if we wish to demonstrate periodicity in $s$ at a fixed time, we have only to demonstrate periodicity in $z$.

We consider a sequence of $n_{b}$ bunches, giving a total charge density of the form

$$
\rho_{\mathrm{tot}}(z)=\sum_{p=-\infty}^{\infty} \sum_{j=1}^{n_{b}} \xi_{j} \rho_{j}\left(z+m_{j} \lambda_{1}+p C\right),
$$

where $\lambda_{1}$ is the wavelength of the main $\mathrm{rf}$ cavity and $C$ is the circumference of the ring. The $m_{j}$ are non-negative integers specifying the filled rf buckets. Without loss of generality, we take $m_{1}=0$; then $m_{j} \leq h-1$, where $h$ is the harmonic number, equal to the maximum number of bunches, and $h \lambda_{1}=C$. We take $\int \rho_{j}(z) d z=1$ and define $\xi_{j}$ as the ratio of the charge in bunch $j$ to the average bunch charge. The leading bunch in a train, having the most negative $z$, has the highest bunch index: $j=n_{b}$.

The bunch profiles $\rho_{j}(z)$ are time independent, since we are concerned with the equilibrium state, and are initially unknown functions to be determined by the condition of equilibrium.

The infinite sequence in (1) is intended to mimic the periodicity of the charge density in a circular storage ring. We have $\rho_{\text {tot }}(z+C)=\rho_{\text {tot }}(z)$, so that at fixed $t$ the density is periodic in $s$ with period $C$. At fixed $s$, it is also periodic in $t$ with period $C / \beta c$. The idealization of supposing that the charge pattern exists for all $t \in(-\infty, \infty)$ is justified, given the large storage times of typical machines.

The total voltage seen by a particle at arbitrary $z$ (at an arbitrary distance from the reference particle) is taken to be

$$
V_{1} \sin \left(k_{1} z+\phi_{0}\right)+V_{r}(z)
$$

where $k_{1}=2 \pi / \lambda_{1}$. In the model to be explored, the induced voltage $V_{r}$ comes only from the lowest mode of the passive harmonic cavity, as excited by the bunch train. The relation of $\phi_{0}$ to the synchronous phase, the phase at which the cavity supplies the mean energy lost per turn, will be discussed presently.

We define $z_{j}$, the argument of the density $\rho_{j}$, as

$$
z_{j}=z+m_{j} \lambda_{1} .
$$

Then by (2) the total voltage as a function of $z_{j}$ is

$$
V_{1} \sin \left(k_{1} z_{j}+\phi_{0}\right)+V_{r}\left(z_{j}-m_{j} \lambda_{1}\right),
$$

since the first term in (2) is periodic in $z$ with period $\lambda_{1}$.

\section{EQUATIONS OF MOTION}

The usual equations of motion for a single particle, subject only to applied rf, describe oscillations in a potential well with the minimum at the location of the synchronous particle. Since the harmonic cavity broadens the well and shifts its minimum, a natural step would be to modify the equation of motion so that it describes oscillations about the shifted minimum. On the other hand, this might be an unnecessary complication if the shift is sufficiently small. The coordinate of the unperturbed problem might provide a perfectly accurate description, even if it is not the distance to the minimum.

We first recall the derivation of the standard equations for a single particle with only applied rf. We first derive difference equations, referring to changes over a full turn, and later replace them by differential equations, since the changes are very small. The salient variable of interest is the phase $\phi$ of the applied rf at the time that the particle crosses the accelerating cavity. 
At the $n$th turn, the rf kick at phase $\phi_{n}$ restores the energy loss $U_{0}$ of the previous turn and also changes the energy of a generic particle from $E_{n}$ to $E_{n+1}$ :

$$
\left(U_{0}+E_{n+1}\right)-E_{n}=e V_{1} \sin \phi_{n} .
$$

The synchronous phase $\phi_{0}$ is that for which the energy supplied is exactly $U_{0}$ :

$$
e V_{1} \sin \phi_{0}=U_{0} .
$$

For stable motion, this angle should be in the second quadrant:

$$
\cos \phi_{0}=-\left(1-\sin ^{2} \phi_{0}\right)^{1 / 2},
$$

with the square root defined to be positive. Defining

$$
(\Delta E)_{n}=E_{n}-E_{0}, \quad(\Delta \phi)_{n}=\phi_{n}-\phi_{0},
$$

where $E_{0}$ is the nominal energy of the ring, we write (5) as

$$
(\Delta E)_{n+1}-(\Delta E)_{n}=e V_{1} \sin \left[(\Delta \phi)_{n}+\phi_{0}\right]-U_{0} .
$$

The change with $n$ of $(\Delta \phi)_{n}$ depends on the revolution frequency, which in turn depends on $(\Delta E)_{n}$, these dependencies being linear to a good approximation. Invoking the definition of the momentum compaction factor $\alpha$, we show in Appendix A that

$$
(\Delta \phi)_{n+1}-(\Delta \phi)_{n}=\alpha k_{1} C \frac{(\Delta E)_{n}}{E_{0}} .
$$

We wish to follow the trajectory of $z$, namely,

$$
z(t)=\beta_{0} c t-s(t),
$$

which is related to the trajectory of $\phi$ as follows:

$$
\begin{aligned}
\frac{\lambda_{1}}{2 \pi}\left[\phi(t)-\phi_{0}\right] & =\text { signed distance to the reference particle } \\
& =z(t),
\end{aligned}
$$

and, hence,

$$
(\Delta \phi)_{n}=k_{1} z_{n} .
$$

The sign in (12) is correct: If $\phi(t)>\phi_{0}$ at time $t$ when the particle arrives at the cavity, it has arrived later than the reference particle, which is to say that $s(t)<\beta_{0} c t$.

Approximating the difference equations (9) and (10) by differential equations, with $d t=T_{0}$ and $\delta=\left(E-E_{0}\right) / E_{0}$, and applying (13), we have

$$
\begin{gathered}
\frac{d \delta}{d t}=\frac{1}{E_{0} T_{0}}\left[e V_{1} \sin \left(k_{1} z+\phi_{0}\right)-U_{0}\right], \\
\frac{d z}{d t}=\alpha \beta_{0} c \delta
\end{gathered}
$$

Here, $T_{0}=C / \beta_{0} c$ is the nominal revolution time of the ring. In replacing (9) by (14), we have equated $T_{0}$ with the time between successive arrivals at the cavity, but this is correct at best in an average sense, because different particles have different revolution times. This approximation is not usually acknowledged in textbook treatments of the problem.

The generalization of (14) and (15) to account for many bunches and the harmonic cavity is obtained by invoking the total voltage (2) and the replacements $z \rightarrow z_{i}-m_{i} \lambda_{1}$, $\delta \rightarrow \delta_{i}$, and, thus,

$$
\frac{d \delta_{i}}{d t}=\frac{1}{E_{0} T_{0}}\left[e V_{1} \sin \left(k_{1} z_{i}+\phi_{0}\right)+e V_{r}\left(z_{i}-m_{i} \lambda_{1}\right)-U_{0}\right],
$$

$$
\frac{d z_{i}}{d t}=\alpha \beta_{0} c \delta_{i}, \quad i=1, \ldots, n_{b} .
$$

For this, we note that the required relation (10) is derived in Appendix A with allowance for the presence of $V_{r}$. The derivation requires $V_{r}(z)=V_{r}(z+C)$, which is assured in the following formalism.

A new feature is that $\phi_{0}$ is no longer the synchronous phase, since the induced voltage $V_{r}$ causes an additional energy increment that must be taken into account. We are nevertheless free to choose $\phi_{0}$ according to (6) and (7), and we shall indeed make that choice. Some nearby value could do as well.

In order to clarify the impact of the shifted synchronous phase, we have also carried out a calculation with the coordinate system shifted accordingly. We conclude that there is no need to work in such a system. This issue is reviewed in Appendix B.

\section{PRIMARY FORMULA FOR THE INDUCED VOLTAGE}

At an arbitrary $z$, the induced voltage from the harmonic cavity will be

$$
\begin{aligned}
V_{r}(z)= & -e N \int_{-\infty}^{\infty} W\left(z-z^{\prime}\right) \rho_{\mathrm{tot}}\left(z^{\prime}\right) d z^{\prime} \\
= & -e N \int_{-\infty}^{\infty} W\left(z-z^{\prime}\right) \\
& \times \sum_{p=-\infty}^{\infty} \sum_{j=1}^{n_{b}} \xi_{j} \rho_{j}\left(z^{\prime}+m_{j} \lambda_{1}+p C\right) d z^{\prime} .
\end{aligned}
$$

Here, $W$ is the wake potential of the cavity, which for sufficiently large $Q$ has the form

$$
W(z)=\frac{\omega_{r} R_{s}}{Q} \theta(z) e^{-k_{r} z / 2 Q} \cos \left(k_{r} z\right) .
$$


In this formula, $\omega_{r}=k_{r} c$ is the circular resonant frequency of the lowest mode of the cavity, $R_{s}$ is its shunt impedance, $Q$ is its quality factor, and $\theta(z)$ is the unit step function, equal to 1 for $z \geq 0$ and 0 otherwise. The $\theta$ function is an expression of causality.

The expression (18) satisfies the obvious requirement that $V_{r}$ be periodic with period $C$. To see that, evaluate $V_{r}(z+C)$ by changing the integration variable to $z^{\prime \prime}=$ $z^{\prime}-C$ and the summation variable to $p^{\prime}=p+1$.

We suppose that the support of any $\rho_{j}(z)$, the region in which it is nonzero, is much less in extent than $\lambda_{1}$, a condition that is satisfied in any ring of interest for this study.

To proceed, it is convenient to translate the variable of integration and reverse the order of integration and summation, so that the formula (18) takes the form

$$
\begin{aligned}
V_{r}(z)= & -e N \int_{-\infty}^{\infty} \sum_{p=-\infty}^{\infty} W\left(z-z^{\prime}+p C\right) \\
& \times \sum_{j=1}^{n_{b}} \xi_{j} \rho_{j}\left(z^{\prime}+m_{j} \lambda_{1}\right) d z^{\prime} .
\end{aligned}
$$

To give an idea of typical parameters for the following work, we list in Table I a tentative set of parameters for ALS-U, the forthcoming upgrade of the Advanced Light Source at Lawrence Berkeley National Laboratory.

\section{EFFECTIVE WAKE POTENTIAL}

The sum over $p$ in (20) can be thought of as an effective wake potential $\mathcal{W}(z)$ which is to be convolved with an effective charge density $\rho(z)$, defined as follows:

\begin{tabular}{|c|c|c|}
\hline Ring circumference & $C$ & $196.5 \mathrm{~m}$ \\
\hline Beam energy & $E_{0}$ & $2 \mathrm{GeV}$ \\
\hline Average bunch current & $I_{\mathrm{avg}}$ & $500 \mathrm{~mA}$ \\
\hline Momentum compaction & $\alpha$ & $2.11 \times 10^{-4}$ \\
\hline Natural energy spread & $\sigma_{\delta}$ & $9.43 \times 10^{-4}$ \\
\hline Natural rms bunch length & $\sigma_{z 0}$ & $3.54 \mathrm{~mm}$ \\
\hline Energy loss per turn & $U_{0}$ & $0.217 \mathrm{MeV}$ \\
\hline Harmonic number & $h$ & 328 \\
\hline Main cavity frequency & $f_{1}$ & $500.417 \mathrm{MHz}$ \\
\hline Main cavity voltage & $V_{1}$ & $0.6 \mathrm{MV}$ \\
\hline $\begin{array}{l}\text { Harmonic cavity } \\
\text { harmonic number }\end{array}$ & 3 & \\
\hline Harmonic cavity frequency & $f_{r}$ & $1501.501 \mathrm{MHz}$ \\
\hline Harmonic cavity detuning & $\delta f=f_{r}-3 f_{1}$ & $0.2502 \mathrm{MHz}$ \\
\hline $\begin{array}{l}\text { Harmonic cavity } \\
\text { shunt impedance }\end{array}$ & $R_{s}$ & $1.39 \mathrm{M} \Omega$ \\
\hline Harmonic cavity quality factor & $Q$ & $2 \times 10^{4}$ \\
\hline
\end{tabular}

TABLE I. Baseline parameters for ALS-U design.
$\mathcal{W}(z)=\sum_{p=-\infty}^{\infty} W(z+p C), \quad \rho(z)=\sum_{j=1}^{n_{b}} \xi_{j} \rho_{j}\left(z+m_{j} \lambda_{1}\right)$

Then the induced voltage may be expressed as

$$
V_{r}(z)=-e N \int_{-\infty}^{\infty} \mathcal{W}\left(z-z^{\prime}\right) \rho\left(z^{\prime}\right) d z^{\prime}
$$

Applying (19) and expanding the cosine by the doubleangle formula, we have

$$
\begin{aligned}
\mathcal{W}(z)= & \frac{\omega_{r} R_{s}}{Q} \sum_{p=-\infty}^{\infty} \theta(z+p C) \\
& \times e^{-k_{r}(z+p C) / 2 Q}\left[\cos \left(k_{r} z\right) \cos \left(p k_{r} C\right)\right. \\
& \left.-\sin \left(k_{r} z\right) \sin \left(p k_{r} C\right)\right]
\end{aligned}
$$

The $\theta$ function requires $p \geq-z / C$, but, since $p$ is an integer, that means

$$
p \geq p_{0}=\lceil-z / C\rceil
$$

where $\lceil x\rceil$ denotes the ceiling of $x$, which is the smallest integer greater than or equal to $x$. Expressing the sine and cosine of $p k_{r} C$ in terms of exponentials, we find that

$$
\begin{aligned}
\mathcal{W}(z)= & \frac{\omega_{r} R_{s}}{Q} e^{-k_{r} z / 2 Q}\left[\cos \left(k_{r} z\right) \operatorname{Re} \zeta\left(k_{r}, z\right)\right. \\
& \left.+\sin \left(k_{r} z\right) \operatorname{Im} \zeta\left(k_{r}, z\right)\right],
\end{aligned}
$$

where

$$
\zeta\left(k_{r}, z\right)=\sum_{p=p_{0}}^{\infty} r^{p h}=r^{p_{0} h} \sum_{p=0}^{\infty} r^{p h}=r^{p_{0} h} \frac{1}{1-r^{h}},
$$

with

$$
r=\exp \left[-k_{r} \lambda_{1}(i+1 / 2 Q)\right]
$$

It is convenient to define real polar variables $\left[\eta\left(k_{r}\right), \psi\left(k_{r}\right)\right]$ such that

$$
\frac{1}{1-r^{h}}=\eta e^{-i \psi}
$$

Then from (26)-(28) we have

$$
\begin{aligned}
& \operatorname{Re} \zeta=\eta e^{-p_{0} k_{r} C / 2 Q} \cos \left(p_{0} k_{r} C+\psi\right) \\
& \operatorname{Im} \zeta=-\eta e^{-p_{0} k_{r} C / 2 Q} \sin \left(p_{0} k_{r} C+\psi\right) .
\end{aligned}
$$


Substituting in (25) and applying the double-angle formula in reverse, we have

$\mathcal{W}(z)=\frac{\omega_{r} R_{s}}{Q} \eta\left(k_{r}\right) \exp \left[-k_{r} \chi(z) / 2 Q\right] \cos \left[k_{r} \chi(z)+\psi\left(k_{r}\right)\right]$,

where

$$
\begin{aligned}
& \chi(z)=z+C\lceil-z / C\rceil=z+n C \\
& \quad \text { for } n C<z \leq(n+1) C, \quad n=\text { integer. }
\end{aligned}
$$

The function $\chi(z)$, plotted in Fig. 1, is periodic with period $C$ and has a sawtooth form, with its value $C$ at jumps defined by the limit from the left. It follows that $\mathcal{W}(z)$ and the induced voltage $V_{r}(z)$ defined by (22) are periodic with period $C$.

In (30), we have an appealing, compact formula for the effective wake potential, which will lead to the induced voltage after a straightforward evaluation of the integral (22). One must keep in mind that the integrand in (22) has a jump at $z=z^{\prime}$ owing to the jump in $\chi(z)$ at $z=0$.

An important feature of $\mathcal{W}(z)$ is its behavior as a function of the detuning parameter $\Delta k / k_{1}$, where for a third-harmonic cavity $\Delta k=k_{r}-3 k_{1}$. Figure 2 shows the real and imaginary parts of the function $\zeta=\eta \exp (-i \psi)$ for a typical case. The function resembles a Lorentzian resonant line form but, in fact, differs substantially from an actual Lorentzian. The half-width of the peak in the real part is roughly $3 /(2 Q)$. Later, we shall find that a true Lorentzian with that half-width occurs in the case of a complete fill with $h$ identical bunches. Since one can show that $\zeta$ approaches a true Lorentzian as $\Delta k$ tends to zero, the maximum of $\eta$ is exactly at $\Delta k=0$.

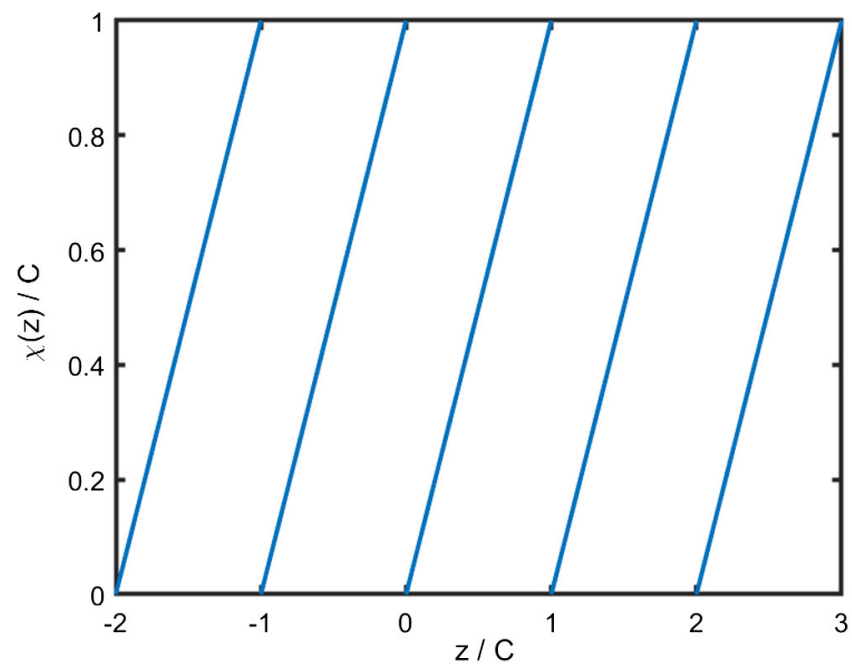

FIG. 1. The sawtooth function $\chi(z)$. Its value at jumps is defined by the limit from the left.

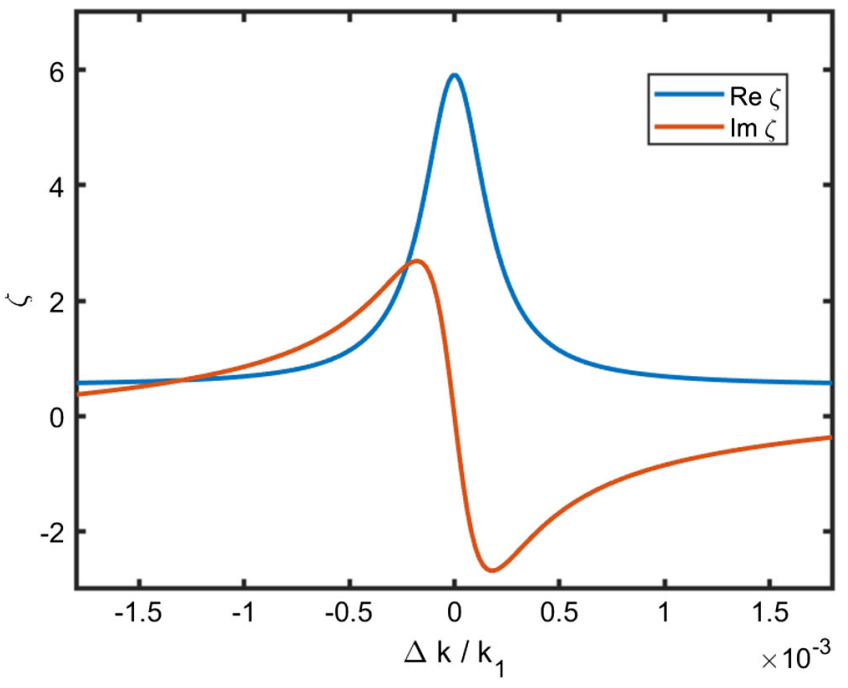

FIG. 2. $\zeta=\eta \exp (-i \psi)$ as a function of the detuning parameter $\Delta k / k_{1}$. The half-width of the peak in the real part is roughly $3 /(2 Q)=0.4 \times 10^{-4}$.

\section{THE INDUCED VOLTAGE}

We are now in a position to compute the induced voltage from (22). The density $\rho$, defined in (21), is zero except for $n_{b}$ isolated peaks, the bunch profiles. We define the interval $\Omega_{i}$ which is to contain the support of the $i$ th bunch, much shorter than the main rf wavelength:

$$
\Omega_{i}=\left\{z \mid-\Sigma \leq z+m_{i} \lambda_{1} \leq \Sigma\right\}, \quad 2 \Sigma \ll \lambda_{1} .
$$

This is just to say that the support in terms of the beam frame coordinate $z_{i}$ is within the region $\left|z_{i}\right| \leq \Sigma$. Note that the elements of $\Omega_{i}$ are close to $z=-m_{i} \lambda_{1}$ and, therefore, decrease with increasing $i$. Because of the stated restriction on $\Sigma$, no two of the $\Omega_{i}$ can intersect:

$$
\Omega_{i} \cap \Omega_{j}=\varnothing, \quad i \neq j .
$$

Some numerical experimentation may be needed to find an appropriate and economical value of $\Sigma$.

We note that $V_{r}(z)$ need be evaluated only at $z$ within the various $\Omega_{i}$, since the collective force enters the dynamics only in those regions, through the Haïssinski or Vlasov equations. Also, $\rho\left(z^{\prime}\right)$ is nonzero only for $z^{\prime}$ in the same sets. It follows that the function $\chi\left(z-z^{\prime}\right)$ in (30) takes on only two values:

$\chi\left(z-z^{\prime}\right)=\left\{\begin{array}{ll}z-z^{\prime}, & z^{\prime}<z, \\ z-z^{\prime}+C, & z^{\prime}>z,\end{array} \quad z \in \Omega_{i}, \quad z^{\prime} \in \Omega_{j}\right.$.

This follows from the fact that $\left|z-z^{\prime}\right|<C$. Regardless of the fill pattern, $\left|z-z^{\prime}\right|$ cannot be greater than a number $C-\lambda_{1}+\mathcal{O}(\Sigma)$. (For instance, if we have only two buckets, $C=2 \lambda_{1}$, and then the distance between a particle in one 
bucket and a particle in the other is $\lambda_{1}$ plus a quantity of order $\Sigma$.) Thus,

$$
\left\lceil-\left(z-z^{\prime}\right) / C\right\rceil= \begin{cases}0, & z^{\prime}<z, \\ 1, & z^{\prime}>z,\end{cases}
$$

which implies (34).

Let us evaluate $V_{r}(z)$ for $z \in \Omega_{i}$ and divide the terms into three groups, those for which $z<z^{\prime}$, those for which $z>z^{\prime}$, and the one "diagonal" term in which both $z<z^{\prime}$ and $z>z^{\prime}$ can occur. Thus,

$$
V_{r}(z)=\frac{-e N \omega_{r} R_{s}}{Q} \eta\left(k_{r}\right)\left[v^{<}(z)+v^{>}(z)+v^{d}(z)\right], \quad z \in \Omega_{i}
$$

where

$$
\begin{gathered}
v^{<}(z)=\sum_{j=1}^{i-1} \xi_{j} \int_{\Omega_{j}} \exp \left[-k_{r}\left(z-z^{\prime}+C\right) / 2 Q\right] \cos \left[k_{r}\left(z-z^{\prime}+C\right)+\psi\left(k_{r}\right)\right] \rho_{j}\left(z^{\prime}+m_{j} \lambda_{1}\right) d z^{\prime}, \\
v^{>}(z)=\sum_{j=i+1}^{n_{b}} \xi_{j} \int_{\Omega_{j}} \exp \left[-k_{r}\left(z-z^{\prime}\right) / 2 Q\right] \cos \left[k_{r}\left(z-z^{\prime}\right)+\psi\left(k_{r}\right)\right] \rho_{j}\left(z^{\prime}+m_{j} \lambda_{1}\right) d z^{\prime}, \\
v^{d}(z)=\xi_{i} \int_{z<z^{\prime}} \exp \left[-k_{r}\left(z-z^{\prime}+C\right) / 2 Q\right] \cos \left[k_{r}\left(z-z^{\prime}+C\right)+\psi\left(k_{r}\right)\right] \rho_{i}\left(z^{\prime}+m_{i} \lambda_{1}\right) d z^{\prime} \\
\quad+\xi_{i} \int_{z>z^{\prime}} \exp \left[-k_{r}\left(z-z^{\prime}\right) / 2 Q\right] \cos \left[k_{r}\left(z-z^{\prime}\right)+\psi\left(k_{r}\right)\right] \rho_{i}\left(z^{\prime}+m_{i} \lambda_{1}\right) d z^{\prime}
\end{gathered}
$$

The sums are regarded as empty when the lower limit exceeds the upper.

In each term of (37) and (38), we change the integration variable to $z_{j}=z^{\prime}+m_{j} \lambda_{1}$, expand the cosine by the doubleangle formula, and recognize the resulting integrals as real and imaginary parts of a Fourier transform at $k_{r}$. Then we find

$$
\begin{aligned}
v^{<}(z)= & \sum_{j=1}^{i-1} \xi_{j} \int_{\Omega_{j}} d z_{j} \rho_{j}\left(z_{j}\right) \exp \left[-k_{r}\left(z-z_{j}+m_{j} \lambda_{1}+C\right) / 2 Q\right] \cos \left[k_{r}\left(z-z_{j}+m_{j} \lambda_{1}+C\right)+\psi\left(k_{r}\right)\right] \\
= & \sum_{j=1}^{i-1} \xi_{j} \exp \left[-k_{r}\left(z+m_{j} \lambda_{1}+C\right) / 2 Q\right] \int_{\Omega_{j}} d z_{j} \exp \left(k_{r} z_{j} / 2 Q\right) \rho_{j}\left(z_{j}\right) \\
& \cdot\left\{\cos \left[k_{r}\left(z+m_{j} \lambda_{1}+C\right)+\psi\left(k_{r}\right)\right] \cos \left(k_{r} z_{j}\right)+\sin \left[k_{r}\left(z+m_{j} \lambda_{1}+C\right)+\psi\left(k_{r}\right)\right] \sin \left(k_{r} z_{j}\right)\right\} \\
= & 2 \pi \sum_{j=1}^{i-1} \xi_{j} \exp \left[-k_{r}\left(z+m_{j} \lambda_{1}+C\right) / 2 Q\right] \\
& \cdot\left\{\cos \left[k_{r}\left(z+m_{j} \lambda_{1}+C\right)+\psi\left(k_{r}\right)\right] \operatorname{Re} \hat{\rho}_{j}\left(k_{r}\right)-\sin \left[k_{r}\left(z+m_{j} \lambda_{1}+C\right)+\psi\left(k_{r}\right)\right] \operatorname{Im} \hat{\rho}_{j}\left(k_{r}\right)\right\},
\end{aligned}
$$

where

$$
\hat{\rho}_{j}\left(k_{r}\right)=\frac{1}{2 \pi} \int_{\Omega_{j}} e^{-k_{r} z(i-1 / 2 Q)} \rho_{j}(z) d z
$$

Carrying out a similar calculation for $v^{>}$, we may write the sum of the two terms as

$$
\begin{aligned}
v^{<}(z)+v^{>}(z)= & 2 \pi \sum_{j=1}^{n_{b}}\left(1-\delta_{i, j}\right) \xi_{j} \exp \left[-k_{r}\left(z+m_{j} \lambda_{1}+\theta_{i-1, j} C\right) / 2 Q\right] \\
& \cdot\left\{\cos \left[k_{r}\left(z+m_{j} \lambda_{1}+\theta_{i-1, j} C\right)+\psi\left(k_{r}\right)\right] \operatorname{Re} \hat{\rho}_{j}\left(k_{r}\right)\right. \\
& \left.-\sin \left[k_{r}\left(z+m_{j} \lambda_{1}+\theta_{i-1, j} C\right)+\psi\left(k_{r}\right)\right] \operatorname{Im} \hat{\rho}_{j}\left(k_{r}\right)\right\}, \quad z \in \Omega_{i},
\end{aligned}
$$


where

$$
\theta_{i, j}= \begin{cases}1, & i \geq j \\ 0, & i<j\end{cases}
$$

The factor $\exp \left(k_{r} z / 2 Q\right)$ is very close to 1 in the present application. In Table I, we have $k_{r} / 2 Q=8 \times 10^{-4} \mathrm{~m}^{-1}$, while $|z|$ is at most about $4 \times 10^{-2} \mathrm{~m}$ in (41). Nevertheless, we shall not replace this factor by 1 , since we expect to apply our formulas to cases with low $Q$ in future work.

The term $v^{d}$ of (39), representing the force on a bunch due to the field that it itself excited, is a bit more complicated. It is not an approximate sinusoid, nor is it expressed in terms of the Fourier transform (41). It is, however, just one of $n_{b}$ terms, not distinguished in magnitude compared to the others, because the high $Q$ of the cavity implies long persistence of the fields excited. The various terms are distinguished principally in phase, through the phase shifts $m_{j} \lambda_{1}$ in their trigonometric arguments. Barring unexpected cancellations, we can regard $v^{d}$ as a minor term and try to determine it iteratively.

\section{COUPLED VLASOV-FOKKER-PLANCK AND HAÏSSINSKI EQUATIONS}

The kinetic equation for the phase space density of the $i$ th bunch is written in terms of the beam frame coordinate $z_{i}=z+m_{i} \lambda_{1}$ of that bunch. It is coupled to the equations for all other bunches through the induced voltage $V_{r}$, which depends on the charge densities of all bunches. In view of the single-particle equations of motion (16) and (17), which define the characteristic curves, the Vlasov-Fokker-Planck equation for the distribution function $f_{i}\left(z_{i}, \delta_{i}, t\right)$ of the $i$ th bunch takes the form

$$
\begin{gathered}
\frac{\partial f_{i}}{\partial t}+\alpha c \delta_{i} \frac{\partial f_{i}}{\partial z_{i}}+\frac{1}{E_{0} T_{0}}\left[e V_{1} \sin \left(k_{1} z_{i}+\phi_{0}\right)\right. \\
\left.+e V_{r}\left(z_{i}-m_{i} \lambda_{1}\right)-U_{0}\right] \frac{\partial f_{i}}{\partial \delta_{i}}=L_{i}^{F P} f_{i} .
\end{gathered}
$$

The Fokker-Planck term on the right-hand side is

$$
L_{i}^{F P} f_{i}=\frac{2}{\omega_{s} t_{d}} \frac{\partial}{\partial \delta_{i}}\left[\delta_{i} f_{i}+\sigma_{\delta}^{2} \frac{\partial f_{i}}{\partial \delta_{i}}\right]
$$

where $\omega_{s}$ is the circular synchrotron frequency and $t_{d}$ is the longitudinal damping time.

We seek an equilibrium in which $\partial f_{i} / \partial t=0$ and $f_{i}$ has the factored Maxwell-Boltzmann form

$$
f_{i}\left(z_{i}, \delta_{i}\right)=\frac{1}{\sqrt{2 \pi} \sigma_{\delta}} \exp \left[-\left(\delta_{i} / \sigma_{\delta}\right)^{2} / 2\right] \rho_{i}\left(z_{i}\right)
$$

Under this hypothesis, the Fokker-Planck term vanishes, and the spatial density $\rho_{i}$ must satisfy

$$
\begin{aligned}
\frac{d \rho_{i}}{d z_{i}}\left(z_{i}\right) & =\frac{1}{\alpha c \sigma_{\delta}^{2} E_{0} T_{0}}\left[e \mathcal{V}\left(z_{i}\right)-U_{0}\right] \rho_{i}\left(z_{i}\right), \\
\mathcal{V}\left(z_{i}\right) & =V_{1} \sin \left(k_{1} z_{i}+\phi_{0}\right)+V_{r}\left(z_{i}-m_{i} \lambda_{1}\right) .
\end{aligned}
$$

By separating variables and integrating, we see that a solution must have the form

$$
\begin{aligned}
\rho_{i}\left(z_{i}\right)= & A_{i}^{-1} \exp \left[\frac{1}{\alpha c \sigma_{\delta}^{2} E_{0} T_{0}} \int_{0}^{z_{i}}\left[e \mathcal{V}(\zeta)-U_{0}\right] d \zeta\right], \\
& -\Sigma \leq z_{i} \leq \Sigma,
\end{aligned}
$$

where $A_{i}$ is a normalization constant, just the integral of the numerator over $[-\Sigma, \Sigma]$.

Noting that $c T_{0}=C$, we introduce the definitions

$\mu=\frac{1}{\alpha \sigma_{\delta}^{2} E_{0} C}, \quad U_{i}\left(z_{i}\right)=-\int_{0}^{z_{i}}\left[e \mathcal{V}(\zeta)-U_{0}\right] d \zeta$.

Recalling (47), we then have (48) expressed as

$$
\begin{aligned}
\rho_{i}\left(z_{i}\right) & =\frac{1}{A_{i}} \exp \left[-\mu U_{i}\left(z_{i}\right)\right], \\
A_{i} & =\int_{-\Sigma}^{\Sigma} \exp \left[-\mu U_{i}(\zeta)\right] d \zeta, \quad-\Sigma \leq z_{i} \leq \Sigma,
\end{aligned}
$$

where

$$
\begin{aligned}
U_{i}\left(z_{i}\right)= & \frac{e V_{1}}{k_{1}}\left[\cos \left(k_{1} z_{i}+\phi_{0}\right)-\cos \phi_{0}\right]+U_{0} z_{i} \\
& +\frac{e^{2} N \omega_{r} R_{s}}{Q} \eta\left(k_{r}\right) \int_{0}^{z_{i}}\left[v^{<}\left(\zeta-m_{i} \lambda_{1}\right)\right. \\
& \left.+v^{>}\left(\zeta-m_{i} \lambda_{1}\right)+v^{d}\left(\zeta-m_{i} \lambda_{1}\right)\right] d \zeta .
\end{aligned}
$$

We refer to $U_{i}$ as the "potential" for the $i$ th bunch, even though it has the dimension of an energy times a length. The system of equations (50) for $i=1, \ldots, n_{b}$ will be called the coupled Haïssinski equations.

\section{MEAN ENERGY TRANSFER IN THE EQUILIBRIUM STATE}

According to (16), the power transferred to a single particle with coordinate $z_{i}$ in the $i$ th bunch is

$$
\begin{aligned}
P_{i}\left(z_{i}\right) & =d E_{i} / d t=E_{0} d \delta_{i} / d t \\
& =\frac{1}{T_{0}}\left[e V_{1} \sin \left(k_{1} z_{i}+\phi_{0}\right)+e V_{r}\left(z_{i}-m_{i} \lambda_{1}\right)-U_{0}\right] .
\end{aligned}
$$

The mean value of the power over the equilibrium distribution is obtained from (47) as 


$$
\int \rho_{i}\left(z_{i}\right) P_{i}\left(z_{i}\right) d z_{i}=\alpha c \sigma_{\delta}^{2} T_{0} \int \frac{d \rho_{i}}{d z_{i}} d z_{i}=0
$$

thus, for every $i$,

$$
\begin{aligned}
U_{0}= & U_{1}+U_{r}=e V_{1} \int \sin \left(k_{1} z+\phi_{0}\right) \rho_{i}(z) d z \\
& +e \int V_{r}\left(z-m_{i} \lambda_{1}\right) \rho_{i}(z) d z .
\end{aligned}
$$

The first term on the right-hand side is the mean energy supplied by the external rf, while the second term, which is negative, represents the mean energy lost to the harmonic cavity per turn. We automatically have energy balance, on the average, in the equilibrium state.

\section{INTEGRAL OF THE INDUCED VOLTAGE}

To express the integral in (51), we define $\mathcal{S}$ and $\mathcal{C}$ as follows:

$$
\begin{aligned}
\mathcal{S}\left(k_{r} z, \phi\right) & =k_{r} \int_{0}^{z} \exp \left(-k_{r} \zeta / 2 Q\right) \cos \left(k_{r} \zeta+\phi\right) d \zeta \\
& =\left\{\frac{\exp \left(-k_{r} z / 2 Q\right)}{1+1 / 4 Q^{2}}\left[\sin \left(k_{r} z+\phi\right)-\frac{1}{2 Q} \cos \left(k_{r} z+\phi\right)\right]\right\}_{0}^{z} \\
& \approx \exp \left(-k_{r} z / 2 Q\right) \sin \left(k_{r} z+\phi\right)-\sin \phi, \\
\mathcal{C}\left(k_{r} z, \phi\right) & =-k_{r} \int_{0}^{z} \exp \left(-k_{r} \zeta / 2 Q\right) \sin \left(k_{r} \zeta+\phi\right) d \zeta \\
& =\left\{\frac{\exp \left(-k_{r} z / 2 Q\right)}{1+1 / 4 Q^{2}}\left[\cos \left(k_{r} z+\phi\right)+\frac{1}{2 Q} \sin \left(k_{r} z+\phi\right)\right]\right\}_{0}^{z} \\
& \approx \exp \left(-k_{r} z / 2 Q\right) \cos \left(k_{r} z+\phi\right)-\cos \phi .
\end{aligned}
$$

The large- $Q$ approximation stated here on the right is not used in our code, since we wish to be set up for later applications with small $Q$.

Applying this in (42), we find

$$
\begin{gathered}
\int_{0}^{z_{i}}\left[v^{<}\left(\zeta-m_{i} \lambda_{1}\right)+v^{>}\left(\zeta-m_{i} \lambda_{1}\right)\right] d \zeta \\
=\frac{2 \pi}{k_{r}} \sum_{j=1}^{n_{b}}\left(1-\delta_{i, j}\right) \xi_{j} \exp \left(-\phi_{i, j} / 2 Q\right) \cdot\left[\mathcal{S}\left(k_{r} z_{i}, \phi_{i, j}+\psi\right) \operatorname{Re} \hat{\rho}_{j}+\mathcal{C}\left(k_{r} z_{i}, \phi_{i, j}+\psi\right) \operatorname{Im} \hat{\rho}_{j}\right], \\
\phi_{i, j}=k_{r}\left[\left(m_{j}-m_{i}\right) \lambda_{1}+\theta_{i-1, j} C\right] .
\end{gathered}
$$

It remains to calculate

$$
\int_{0}^{z_{i}} v^{d}\left(\zeta-m_{i} \lambda_{1}\right) d \zeta
$$

with $v^{d}$ from (39). After changing the integration variable in (39) to $u=z^{\prime}+m_{i} \lambda_{1}$, we have

$$
\begin{aligned}
v^{d}\left(\zeta-m_{i} \lambda_{1}\right)= & \xi_{i} \int_{-\Sigma}^{\zeta} \exp \left[-k_{r}(\zeta-u) / 2 Q\right] \cos \left[k_{r}(\zeta-u)+\psi\right] \rho_{i}(u) d u \\
& +\xi_{i} \int_{\zeta}^{\Sigma} \exp \left[-k_{r}(\zeta-u+C) / 2 Q\right] \cos \left[k_{r}(\zeta-u+C)+\psi\right] \rho_{i}(u) d u .
\end{aligned}
$$

We can avoid the double integral in (59) through an integration by parts. After applying the double-angle formula to the cosine, one of the terms comprising (59) takes the form

$$
\xi_{i} \int_{0}^{z_{i}} \exp \left(-k_{r} \zeta / 2 Q\right) \cos \left(k_{r} \zeta+\psi\right) \int_{-\Sigma}^{\zeta} \exp \left(k_{r} u / 2 Q\right) \cos \left(k_{r} u\right) \rho_{i}(u) d u
$$


Now in a partial integration the factor $\exp \left(-k_{r} \zeta / 2 Q\right) \cos \left(k_{r} \zeta+\psi\right)$ is integrated by applying (55), while the $u$ integral is differentiated. Proceeding similarly with the other terms, we eliminate all double integrals.

\section{SOLUTION OF COUPLED HAÏSSINSKI EQUATIONS BY NEWTON'S METHOD}

Let us multiply (50) by $\exp \left(k_{r} z_{i} / 2 Q\right)$ and then take the Fourier transform, as in (41). This yields

$$
\hat{\rho}_{i}\left(k_{r}\right)-\frac{1}{2 \pi A_{i}} \int_{-\Sigma}^{\Sigma} \exp \left[-k_{r} z(i-1 / 2 Q)-\mu U_{i}(z)\right] d z=0, \quad i=1, \ldots, n_{b},
$$

where $A_{i}$ is defined in (50) and

$$
\begin{aligned}
U_{i}(z)= & \frac{e V_{1}}{k_{1}}\left[\cos \left(k_{1} z+\phi_{0}\right)-\cos \phi_{0}\right]+U_{0} z+\frac{e^{2} N \omega_{r} R_{s} \eta}{Q}\left[\int_{0}^{z} v^{d}\left(\zeta-m_{i} \lambda_{1}\right) d \zeta\right. \\
& \left.\times \frac{2 \pi}{k_{r}} \sum_{j=1}^{n_{b}}\left(1-\delta_{i, j}\right) \xi_{j} \exp \left(-\phi_{i, j} / 2 Q\right) \cdot\left[\mathcal{S}\left(k_{r} z_{i}, \phi_{i, j}+\psi\right) \operatorname{Re} \hat{\rho}_{j}+\mathcal{C}\left(k_{r} z_{i}, \phi_{i, j}+\psi\right) \operatorname{Im} \hat{\rho}_{j}\right]\right] .
\end{aligned}
$$

If the diagonal term in $v^{d}$ were known, the real and imaginary parts of (62) would constitute $2 n_{b}$ equations in the $2 n_{b}$ unknowns $\operatorname{Re} \hat{\rho}_{j}$ and $\operatorname{Im} \hat{\rho}_{j}$. Defining a notation for the diagonal term,

$$
u_{i}^{d}(z)=\int_{0}^{z} v^{d}\left(\zeta-m_{i} \lambda_{1}\right) d \zeta
$$

we write (62) more briefly as

$$
F\left(\hat{\rho}, u^{d}\right)=0
$$

where $F$ and $\hat{\rho}$ are complex column vectors with $n_{b}$ components, in which

$$
\begin{aligned}
F_{i}\left(\hat{\rho}, u^{d}\right)= & A_{i} \hat{\rho}_{i}-\frac{1}{2 \pi} \int_{-\Sigma}^{\Sigma} \exp \left[-k_{r} z(i-1 / 2 Q)\right. \\
& \left.-\mu U_{i}\left(z, \hat{\rho}, u^{d}\right)\right] d z .
\end{aligned}
$$

For given $u^{d}$, we try to solve (65) by the matrix form of Newton's method, namely,

$$
\begin{gathered}
F\left(\hat{\rho}^{(n)}, u^{d}\right)+\frac{\partial F\left(\hat{\rho}^{(n)}, u^{d}\right)}{\partial \operatorname{Re} \hat{\rho}} \operatorname{Re}\left(\hat{\rho}^{(n+1)}-\hat{\rho}^{(n)}\right) \\
+\frac{\partial F\left(\hat{\rho}^{(n)}, u^{d}\right)}{\partial \operatorname{Im} \hat{\rho}} \operatorname{Im}\left(\hat{\rho}^{(n+1)}-\hat{\rho}^{(n)}\right)=0 .
\end{gathered}
$$

Here, $(\partial F / \partial \operatorname{Re} \hat{\rho}, \partial F / \partial \operatorname{Im} \hat{\rho})$ are complex matrices with elements $\left(\partial F_{i} / \partial \operatorname{Re} \hat{\rho}_{j}, \partial F_{i} / \partial \operatorname{Im} \hat{\rho}_{j}\right)$. That is, we linearize $F$ about iterate $\hat{\rho}^{(n)}$ to define the update $\hat{\rho}^{(n+1)}$ by (67). In (67), we have $2 n_{b}$ real linear equations to solve for the increment $\hat{\rho}^{(n+1)}-\hat{\rho}^{(n)}$.
Lacking any better choice, we begin the process with $\hat{\rho}^{(0)}$ obtained from Gaussians, all with the nominal bunch length:

$$
\begin{aligned}
\hat{\rho}_{i}^{(0)}\left(k_{r}\right)= & \frac{1}{(2 \pi)^{3 / 2} \sigma_{z}} \int_{-\Sigma}^{\Sigma} \exp \left[k_{r} z(-i+1 / 2 Q)\right. \\
& \left.-\left(z / \sigma_{z}\right)^{2} / 2\right] d z, \quad i=1, \ldots, n_{b} .
\end{aligned}
$$

Note that we could not use the direction-independent complex derivative $\partial / \partial \hat{\rho}_{i}$, since $U_{i}$ is not an analytic function of $\hat{\rho}_{i}$, being always real.

To account for the diagonal term, we adopt the simple device of computing $u^{d}$ in (67) from the previous Newton iterate. That procedure yields a convergent scheme and shows that the contribution of the diagonal term is negligible, at least in the present case of a high- $Q$ cavity. If our scheme is later applied to a low- $Q$ case, a more sophisticated method might be needed to determine $v^{d}$.

\section{EXPRESSION OF THE JACOBIAN MATRIX}

Since the exponent $U_{i}$ is linear in the unknowns $\hat{\rho}_{j}$, it is not difficult to write down the Jacobian, the matrix of the partial derivatives that appears in (67). One must not forget the derivatives of $A_{i}$, which are essential to ensure that the final $\rho_{i}\left(z_{i}\right)$ are automatically normalized to have unit integral. The complete $2 n_{b} \times 2 n_{b}$ Jacobian in block matrix form is

$$
\left[\begin{array}{ll}
\partial \operatorname{Re} F / \partial \operatorname{Re} \hat{\rho} & \partial \operatorname{Re} F / \partial \operatorname{Im} \hat{\rho} \\
\partial \operatorname{Im} F / \partial \operatorname{Re} \hat{\rho} & \partial \operatorname{Im} F / \partial \operatorname{Im} \hat{\rho}
\end{array}\right]
$$

where 


$$
\begin{aligned}
& \frac{\partial \operatorname{Re} F_{i}}{\partial \operatorname{Re} \hat{\rho}_{j}}= A_{i} \delta_{i j}+\mu \int\left[\operatorname{Re} \hat{\rho}_{i}+\frac{1}{2 \pi} \cos \left(k_{r} z\right) e^{k_{r} z / 2 Q}\right] \\
& \times e^{-\mu U_{i}(z)} a_{i j}(z) s_{i j}(z) d z, \\
& \frac{\partial \operatorname{Re} F_{i}}{\partial \operatorname{Im} \hat{\rho}_{j}}= \mu \int\left[\operatorname{Re} \hat{\rho}_{i}+\frac{1}{2 \pi} \cos \left(k_{r} z\right) e^{k_{r} z / 2 Q}\right] \\
& \times e^{-\mu U_{i}(z)} a_{i j}(z) c_{i j}(z) d z, \\
& \frac{\partial \operatorname{Im} F_{i}}{\partial \operatorname{Re} \hat{\rho}_{j}}=\mu \int\left[\operatorname{Im} \hat{\rho}_{i}-\frac{1}{2 \pi} \sin \left(k_{r} z\right) e^{k_{r} z / 2 Q}\right] \\
& \times e^{-\mu U_{i}(z)} a_{i j}(z) s_{i j}(z) d z,
\end{aligned}
$$

$$
\begin{aligned}
\frac{\partial \operatorname{Im} F_{i}}{\partial \operatorname{Im} \hat{\rho}_{j}}= & A_{i} \delta_{i j}+\mu \int\left[\operatorname{Im} \hat{\rho}_{i}-\frac{1}{2 \pi} \sin \left(k_{r} z\right) e^{k_{r} z / 2 Q}\right] \\
& \times e^{-\mu U_{i}(z)} a_{i j}(z) c_{i j}(z) d z,
\end{aligned}
$$

$$
F[\hat{\rho}(I), I]=0
$$

and differentiate with respect to $I$ to obtain

$$
\frac{\partial F}{\partial \operatorname{Re} \hat{\rho}} \frac{d \operatorname{Re} \hat{\rho}}{d I}+\frac{\partial F}{\partial \operatorname{Im} \hat{\rho}} \frac{d \operatorname{Im} \hat{\rho}}{d I}+\frac{\partial F}{\partial I}=0
$$

The solution of this linear system for $d \hat{\rho} / d I$ affords the linear extrapolation

$$
\hat{\rho}(I+\Delta I)=\hat{\rho}(I)+\frac{d \hat{\rho}(I)}{d I} \Delta I .
$$

The extrapolation is not a costly step, since the Jacobian matrix in (78) is already known from the previous Newton iteration.

It is helpful to redefine the unknown as $\tilde{\rho}=I \hat{\rho}$, so that the current appears linearly in the transformed version of (77), namely,

$$
\begin{aligned}
\tilde{F}_{i}(\tilde{\rho}, I)= & A_{i} \tilde{\rho}_{i}-\frac{I}{2 \pi} \int_{-\Sigma}^{\Sigma} \exp \left[-k_{r} z(i-1 / 2 Q)\right. \\
& \left.-\mu \tilde{U}_{i}(z, \tilde{\rho})\right] d z=0 .
\end{aligned}
$$

Now even the right-hand side of the equation to be solved, $-\partial \tilde{F} / \partial I$, is an integral already computed during the previous Newton iteration.

Our continuation procedure is an example of a general method for solving nonlinear problems, called path following or executing a homotopy [36]. One follows a known or easily computed solution as a function of a parameter, which could be multidimensional. The continuation could stall or display a bifurcation if a singularity of the Jacobian were encountered.

\section{NUMERICAL RESULTS FOR PARAMETERS OF ALS-U}

\section{A. Complete train without gaps}

We first present results for a complete train without gaps, for which $n_{b}=h=328$, at the nominal current of $500 \mathrm{~mA}$. The calculation starts at a current of $150 \mathrm{~mA}$ and proceeds to the desired current in three equal increments, by means of the algorithm of the previous section. The convergence criterion for a Newton iteration is in terms of a sum of normalized residuals of the equation [Eq. (65)] that is to be satisfied:

$$
\epsilon=\sum_{i=1}^{n_{b}} \frac{\left|\operatorname{Re} F_{i}\right|+\left|\operatorname{Im} F_{i}\right|}{A_{i}\left(\left|\operatorname{Re} \hat{\rho}_{i}\right|+\left|\operatorname{Im} \hat{\rho}_{i}\right|\right)} .
$$

With convergence defined by $\epsilon<10^{-12}$, the Newton iterations converge in at most seven steps, and the CPU time for the whole calculation is $25 \mathrm{~s}$ on a laptop PC. 


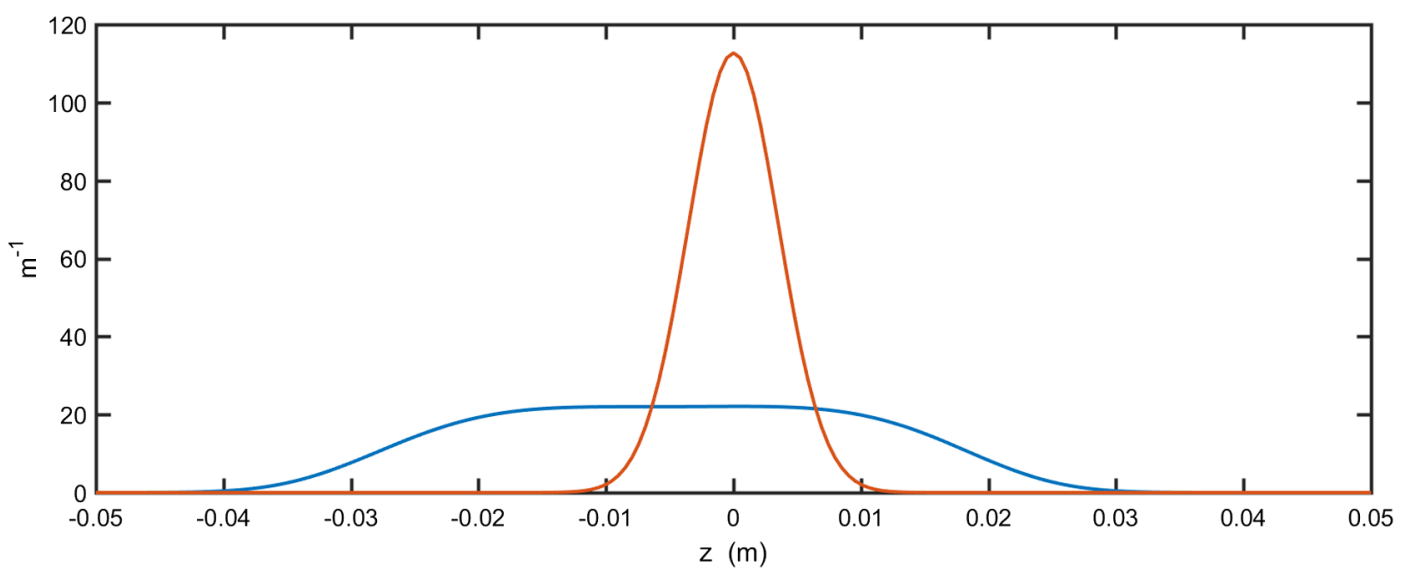

FIG. 3. Charge density for complete fill at $500 \mathrm{~mA}$ (blue curve), with rms width $\sigma=4.08 \sigma_{z 0}$ and centroid $\langle z\rangle=-4.74 \mathrm{~mm}$. Gaussian solution at a low current (red curve) with $\sigma_{z 0}=3.54 \mathrm{~mm}$.

The program is a serial code in FORTRAN, using the Intel Math Kernel Library for the linear algebra. As is typical of Newton's method, the convergence is very rapid at the last steps, with $\epsilon$ bounded by $\epsilon^{2}$ of the previous step. The number of mesh points for the integrals on $z$ was 201, and the mesh covers $\left[-6 \sigma_{z 0}, 6 \sigma_{z 0}\right]$. The solution with twice as many points is the same to graphical accuracy and takes twice the CPU time.

Figure 3 shows the resulting charge density of 328 identical bunches, for the parameters of Table I, together with the Gaussian low-current solution having the natural bunch width $\sigma_{z 0}=3.54 \mathrm{~mm}$. At full current, the harmonic cavity increases the rms width to $4.08 \sigma_{z 0}$ and causes a centroid displacement of $-4.74 \mathrm{~mm}$.

The shunt impedance and detuning in Table I were chosen to maximize the bunch lengthening at the nominal current while keeping a flattop in the density. At higher impedance or lower detuning, one can achieve a larger bunch lengthening but at the expense of getting a density with two maxima. The bunch in this situation is sometimes described as being "overstretched". Figure 4 shows the effect of decreasing the detuning and lists the corresponding rms bunch lengths.

It is important to note that all bunch forms turn out to be the same, merely by putting $n_{b}=h$, even though the equations contain no explicit constraint that they be the same. This is gratifying and as it should be by physical intuition, but the mathematical mechanism for it to happen is somewhat obscure.

\section{B. Train with a single gap}

Next, we consider a train with a single gap of 44 empty buckets, thus 284 filled buckets in a row. The average current, i.e., the total charge divided by the revolution time, is taken to be the same as before, corresponding to the individual bunch charge being larger by a factor of $328 / 284$. With an initial average current of $150 \mathrm{~mA}$, increased to $500 \mathrm{~mA}$ in three steps, the convergence is even better than in the previous example.

In Fig. 5, we show representative bunch forms near the front, middle, and end of the train. Each bunch is given as a function of its beam frame coordinate $z_{j}=z+m_{j} \lambda_{1}$.

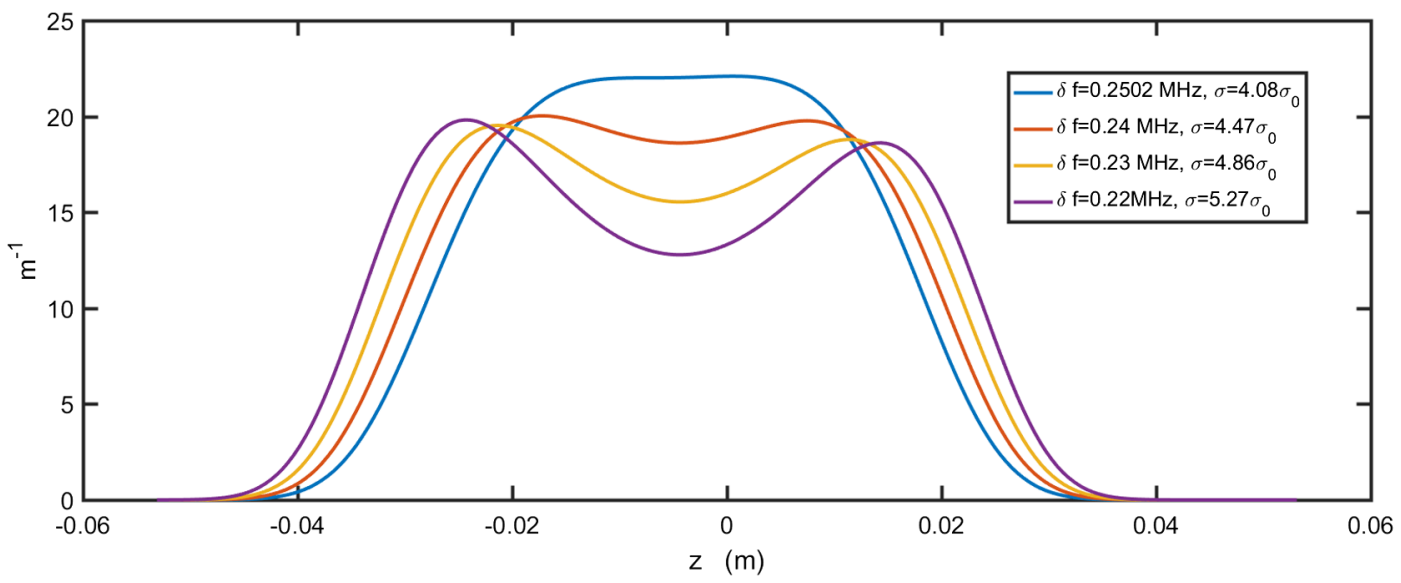

FIG. 4. Charge density for complete fill at $500 \mathrm{~mA}$, for decreasing values of the detuning $\delta f=f_{r}-3 f_{1}=(0.2502,0.24$, $0.23,0.22) \mathrm{MHz}$, with corresponding bunch lengths $\sigma=(4.08,4.47,4.86,5.27) \sigma_{0}$. 


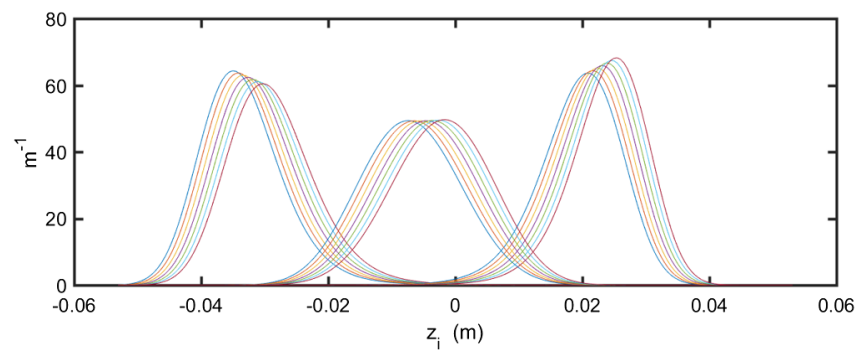

FIG. 5. Results for a single gap of 44 empty buckets. On the right, seven bunches within the first 24 ; in the middle, seven bunches within the middle 24; on the left, seven bunches within the last 24 .
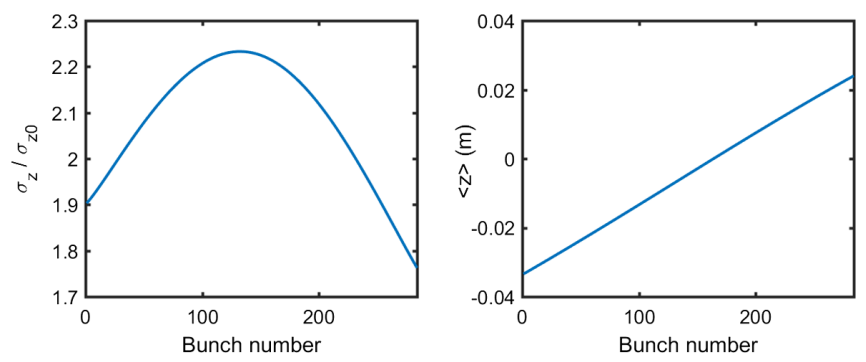

FIG. 6. Results for a single gap of 44 empty buckets. In the left plot, the ratio of rms bunch length to the natural bunch length vs bunch number, head of the train on the right. In the right plot, the corresponding graph of the centroid position.

There is much less bunch lengthening than in the complete fill and a large centroid shift varying linearly along the train. In Fig. 6 (left), we show the variation of bunch length (divided by the natural bunch length) along the train, while Fig. 6 (right) shows the variation of the centroid position. The maximum centroid shift is 7 times larger than in the complete fill.

\section{Train with distributed gaps}

The sharp reduction in bunch lengthening induced by a single gap leads to the idea of distributing the empty buckets around the ring as much as possible [37]. This has a chance of resembling more closely the complete fill. For ALS-U, the minimum acceptable gap consists of four empty buckets, since a gap of $10 \mathrm{~ns}$ is required to accommodate the rise and fall times of the fast kicker that does on-axis injection from the accumulator ring. With such gaps, we need nine trains of 26 bunches and two of 25 to account for 328 buckets in total: $9 \times 26+2 \times 25+11 \times 4=328$.

We consider case C2 of Ref. [37], in which the two trains of 25 are as far apart as possible. This was found to be slightly more helpful than putting those two side by side. Figure 7 shows the result for six bunches out of a train of 26 , including the initial and final bunches. Figure 8 shows the ratio of the rms bunch length to the natural bunch length vs bunch number, while Fig. 9 displays the centroid vs

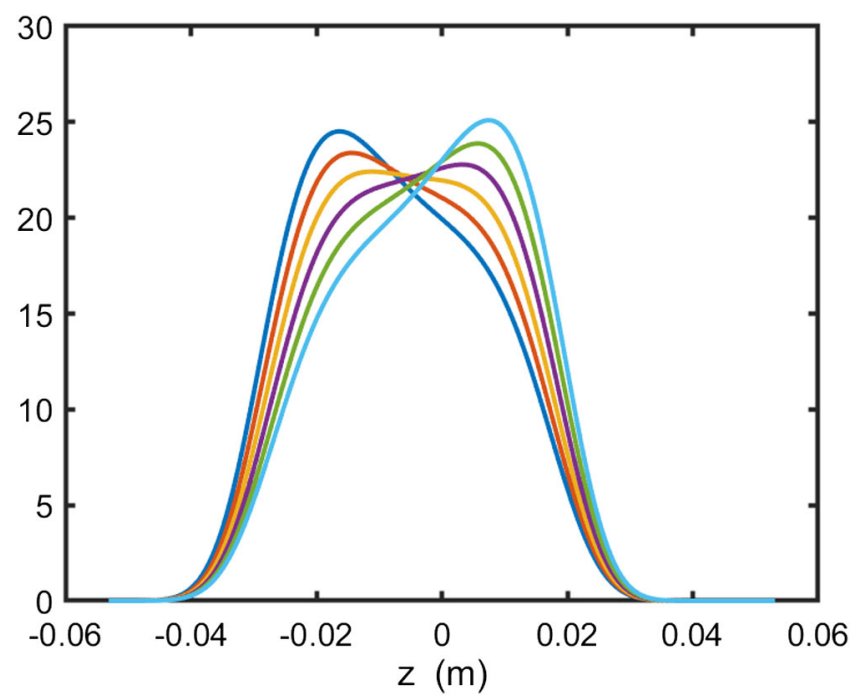

FIG. 7. Results for distributed gaps, each consisting of four empty buckets. Plots of six bunches out of a train of 26 .

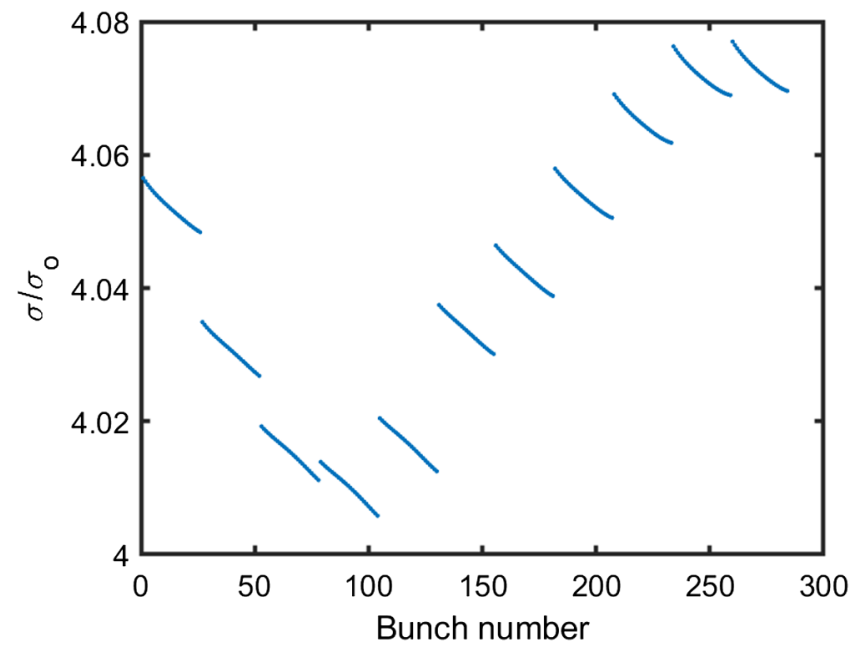

FIG. 8. Ratio of bunch length to natural bunch length vs bunch number, with distributed gaps.

bunch number. Fortunately, the average bunch lengthening now has a value near the case of the complete fill. Furthermore, the big centroid displacement of the singlegap case is gone. There is a small and linear centroid displacement along each subtrain, but its magnitude is similar to that of the complete fill.

Although maximal distribution of the gaps is a step in the right direction, it leaves us with a strong variation of bunch form along the train and some highly skewed charge distributions. We should then look for further means to imitate the complete fill as much as possible.

\section{Guard bunches to compensate the damage from gaps}

If bunches at or near the ends of a train are given greater charge, enough to equal the missing charge due to the gap, 


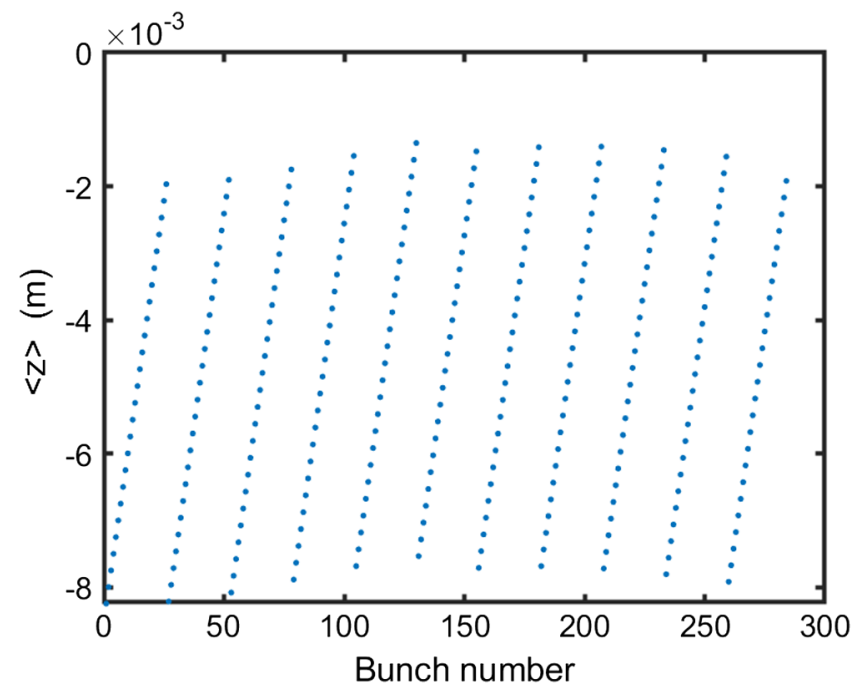

FIG. 9. Centroid vs bunch number, with distributed gaps.

the inner bunches may feel less perturbation from the gap. This idea was advanced by Byrd et al. [31] in 2002 and reinvented at the Argonne APS in 2017 [38]. These enhanced bunches have come to be called guard bunches. We first modify the setup of the previous subsection by increasing the charge in the first and last bunches of each of the 11 subtrains by a factor of 3 [for example, so that $\xi(1)=\xi(25)=3$ ], thus trying to compensate for four missing bunches. (Of course, it is no longer necessary to increase the total charge to compare with the complete fill.) Remarkably, this causes all of the inner bunches to be identical to graphical accuracy, with a perfect flattop as in

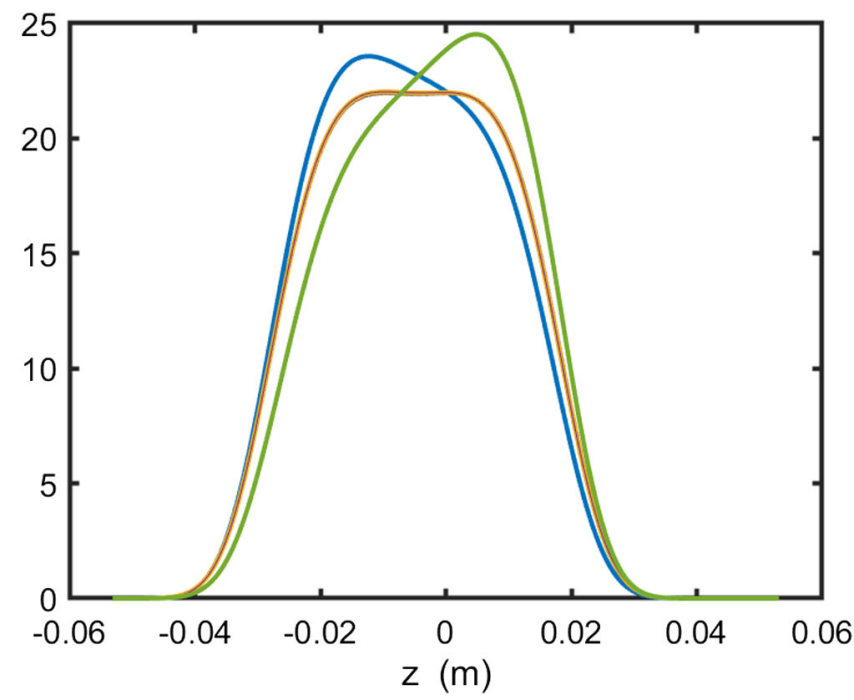

FIG. 10. A train of 26 with a guard bunch at its beginning (on the right) and at its end (on the left). The guard bunches have 3 times the charge of the inner bunches, to compensate for the charge missing in four gaps. The 24 inner bunches (in red) are nearly identical.

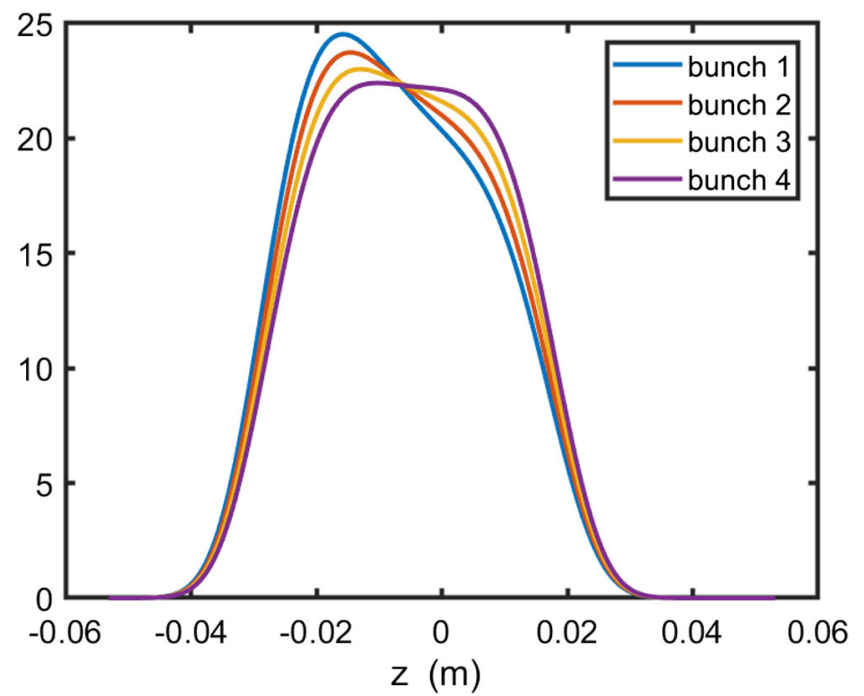

FIG. 11. The four guard bunches at the end of a train of 26, each with $\xi=1.5$. These lean forward.

the complete fill. Only the guard bunches differ from this pattern, as is seen in Fig. 10.

Such highly intense guard bunches could suffer a microwave instability, or have a reduced lifetime, or be undesirable for their impact on the synchrotron light pattern. It therefore becomes interesting to distribute the guard charge over several bunches. As an example, we take four guard bunches at the beginning of the train and four at the end, each with 50\% more charge than the inner bunches $(\xi=1.5)$. As is shown in Figs. 11-13, the inner bunches again are flattopped, while there is a gradual transition in the guard sequence from the end bunch form to the inner.

Rather than a uniform distribution of charge in the guard segment, one could try some kind of taper, for instance, a power law with an arbitrary exponent:

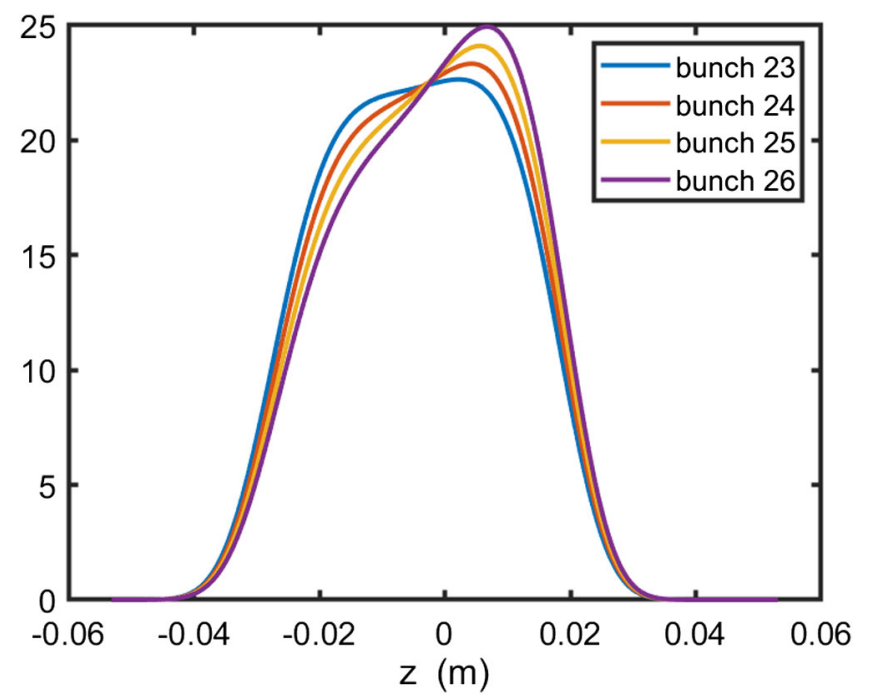

FIG. 12. The four guard bunches at the beginning of a train of 26 , each with $\xi=1.5$. These lean backward. 


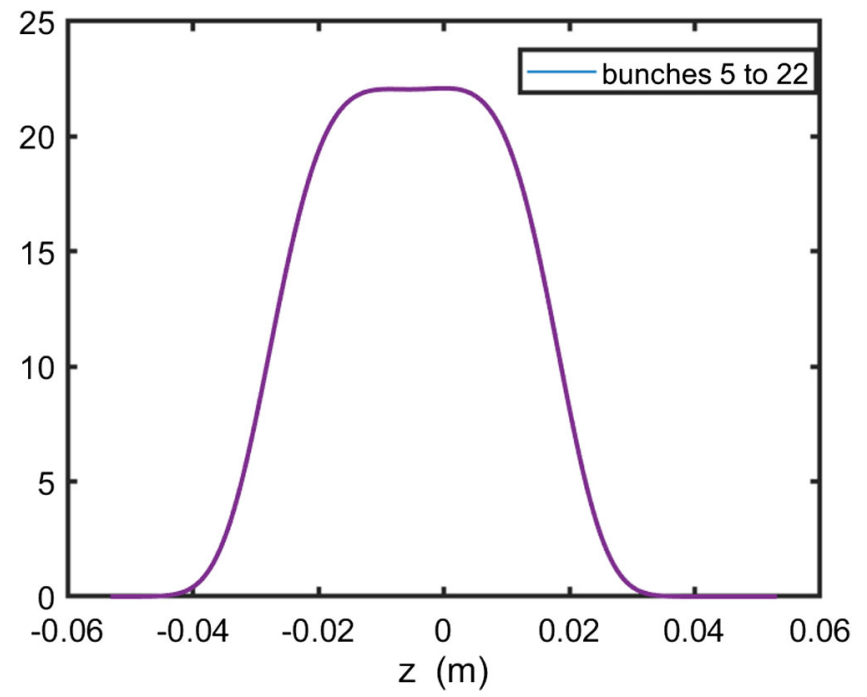

FIG. 13. The 18 normal bunches in the middle of the train, each with $\xi=1$.

$$
\begin{aligned}
\xi(j) & =1+a j^{-b}, \quad j=1,2, \ldots n_{g}, \\
\xi(j) & =1+a\left(n_{t}+1-j\right)^{-b}, \\
j & =n_{t}, n_{t}-1, \ldots, n_{t}-n_{g}+1,
\end{aligned}
$$

for a train of $n_{t}$ bunches with $n_{g}$ guard bunches at either end. We might try a sharply peaked distribution with large $b$ in order to imitate the case of a single guard bunch but with less peak charge. For instance, putting $b=1.7$ and $n_{g}=13$ in a train with $n_{t}=26$, we get the result in Fig. 14. The bunch population $\xi(j)$ of (82) is plotted in Fig. 15. With $30 \%$ less charge in the end bunches, we get a pattern very similar to that of a single guard bunch, in that most of the interior bunches are close to flattopped and have markedly

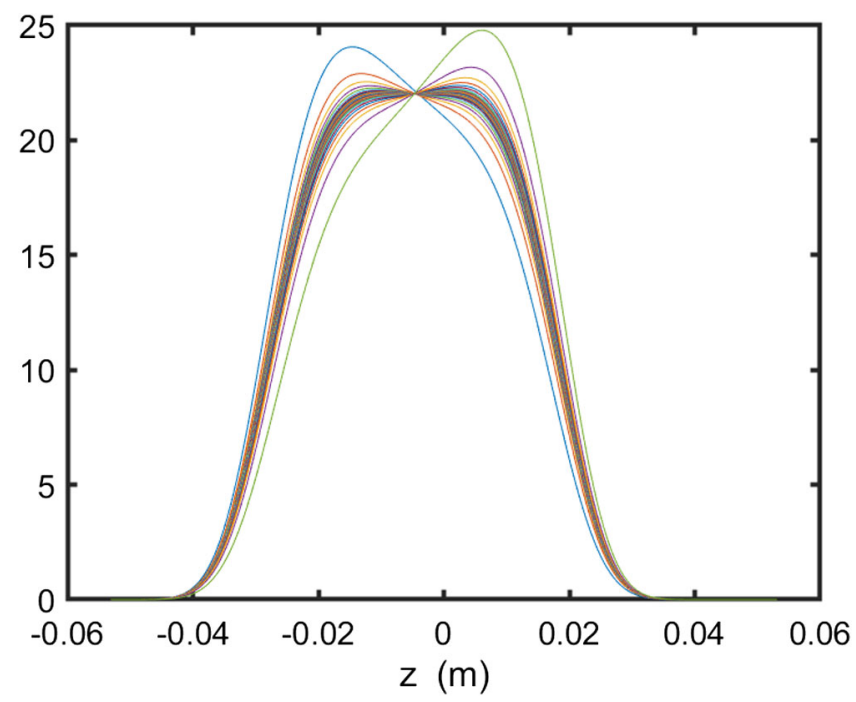

FIG. 14. A train of 26 with the charge distribution of Eq. (82), $b=1.7$, and $n_{g}=13$.

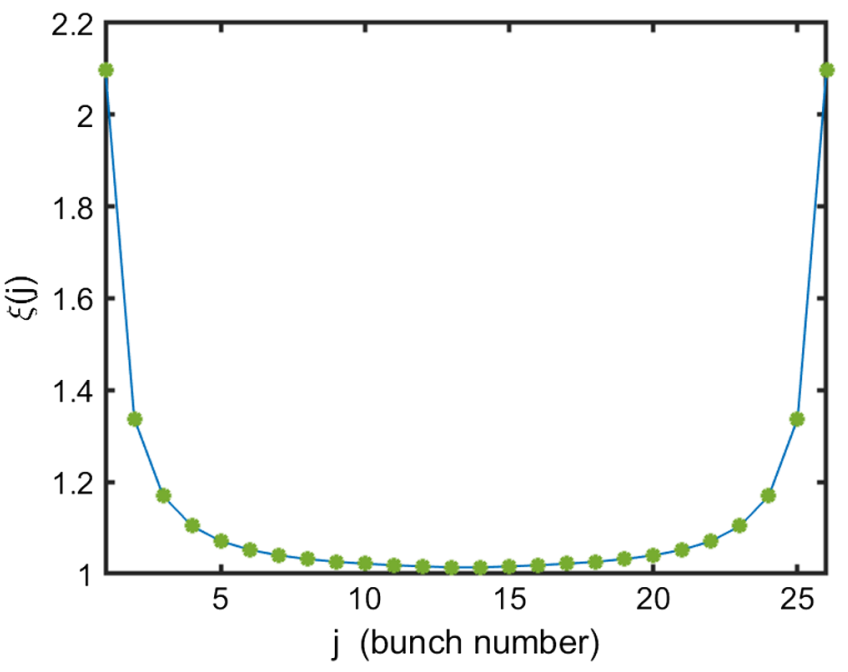

FIG. 15. The bunch population factor $\xi(j)$ of Eq. (82) for $b=$ 1.7 and $n_{g}=13$.

less charge than the four guard bunches with uniform population as considered above (Figs. 11-13).

\section{E. Comparison to a macroparticle simulation}

We have applied the code ELEGANT [39] to make a macroparticle simulation for comparison to results of the present method. This was part of an exploration of parameter space, and the parameters are different from those of Table I in the following choices:

$$
\begin{aligned}
\alpha & =2.07 \times 10^{-4}, \quad \sigma_{\delta}=1.14 \times 10^{-3}, \\
\sigma_{z 0} & =4.43 \mathrm{~mm}, \quad U_{0}=3.29 \times 10^{5} \mathrm{eV}, \\
R_{s} & =10^{6} \Omega, \quad Q=1.67 \times 10^{4}, \\
\delta f & =2.27 \times 10^{5} \mathrm{~Hz} .
\end{aligned}
$$

Also, in (16) we take $e V \sin \phi_{0}=(9 / 8) U_{0}$, following Eq. (B10) in Ref. [27]. The simulation used the cavity wakefield description provided by ELEGANT and was done with 10000 macroparticles per bunch. The fill pattern is that of Sec. XIII C, with 284 bunches and distributed gaps of four buckets each.

Results are shown in Figs. 16-18. The agreement is good enough both to provide a check on our semianalytic scheme and to affirm the viability of a macroparticle simulation. It is extremely surprising that the agreement is much better on the tails of the distribution than in the core.

\section{INCREASE IN THE TOUSCHEK LIFETIME}

Following Refs. [3,40], we note that in Piwinski's analysis [1] the Touschek lifetime is inversely proportional to the integral of the square of the longitudinal charge density. Under bunch lengthening, the lifetime should then increase by a factor 


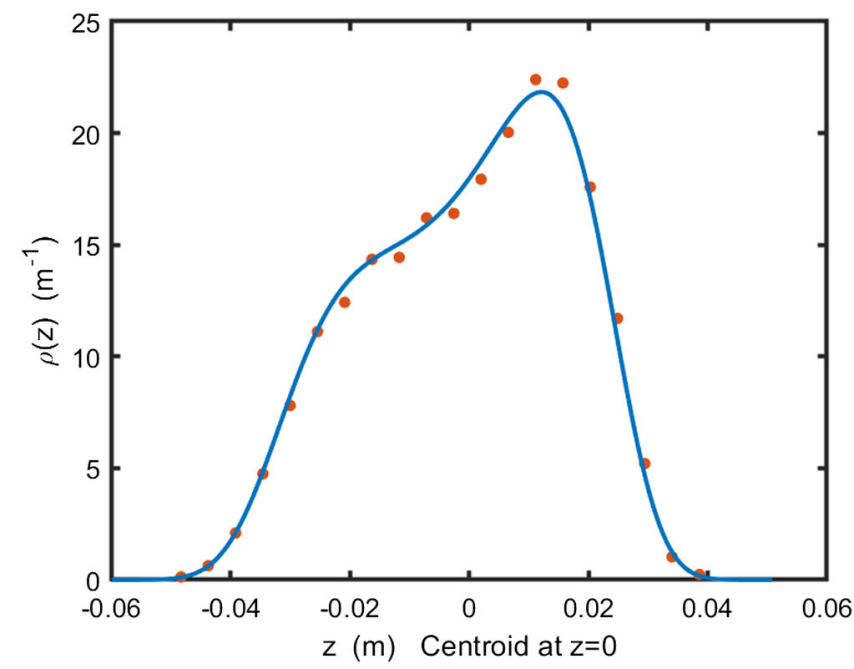

FIG. 16. $\rho(z)$ of the first bunch in a train of 26 , by macroparticle simulation (red dots) and Haïssinski solution (blue curve).

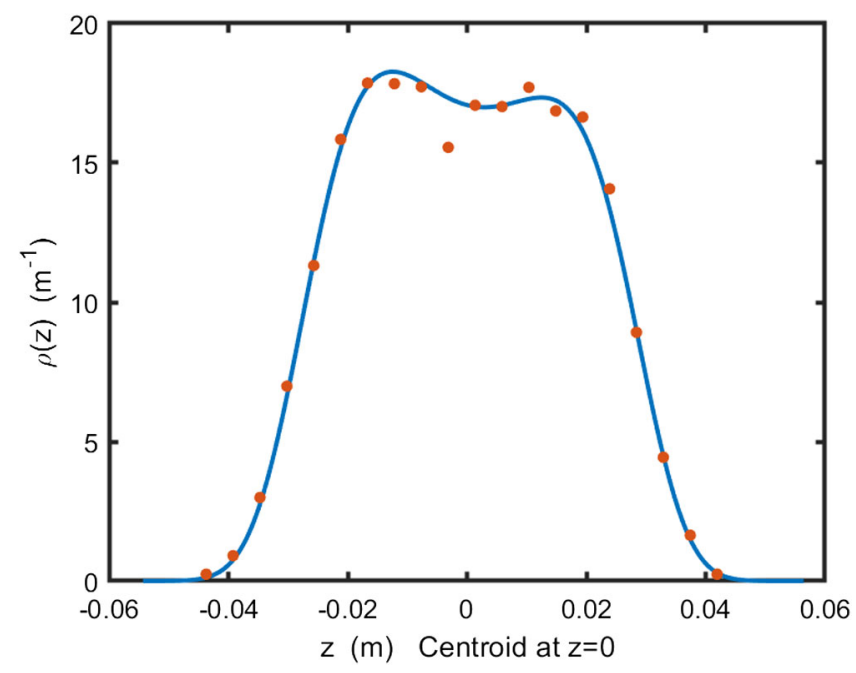

FIG. 17. $\rho(z)$ of the last bunch in a train of 26, by macroparticle simulation (red dots) and Haïssinski solution (blue curve).

$$
R=\frac{\tau}{\tau_{0}} \approx \frac{\int \rho_{0}^{2}(z) d z}{\int \rho^{2}(z) d z}
$$

where $\rho$ and $\rho_{0}$ are the charge densities with and without, respectively, the action of the harmonic cavity. The approximation in (84) consists of not including the ratio of momentum acceptances for the two cases, which Byrd and Georgsson [3] judge to be of the order of 1. Taking $\rho_{0}$ to be a Gaussian with the nominal (zero current) bunch length, we compute $R$ by (84) for the case of distributed gaps, as in Sec. XIII C, without guard bunches.

Figure 19 shows the variation of $R$ along a train of 26, for various choices of the cavity detuning to demonstrate the effect of overstretching. At the nominal detuning of $\delta f=250.2 \mathrm{kHz}$, there is an almost uniform increase of

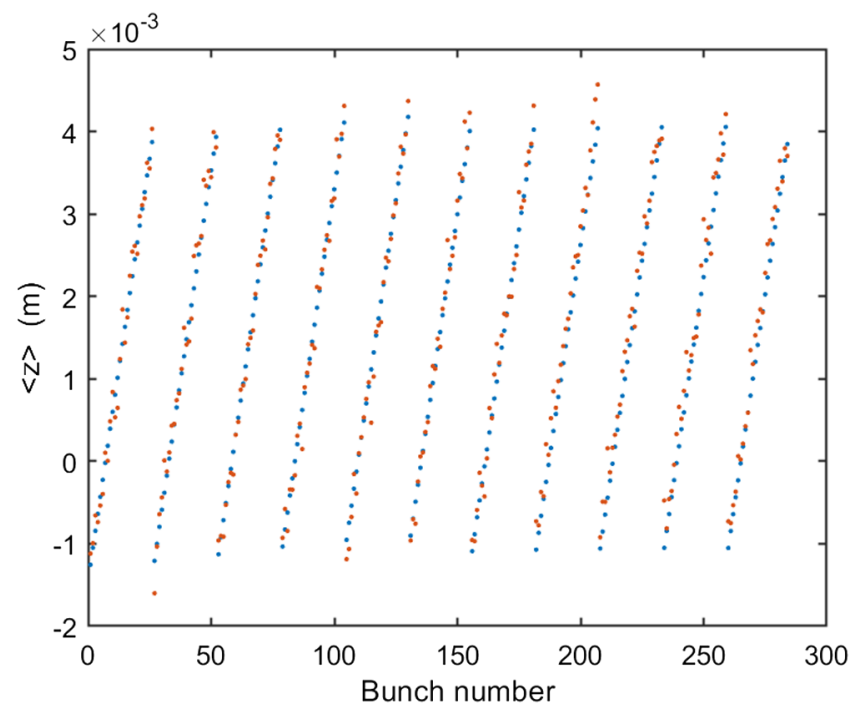

FIG. 18. Centroids of a train with distributed gaps, by macroparticle simulation (red dots) and Haïssinski solution (blue dots).

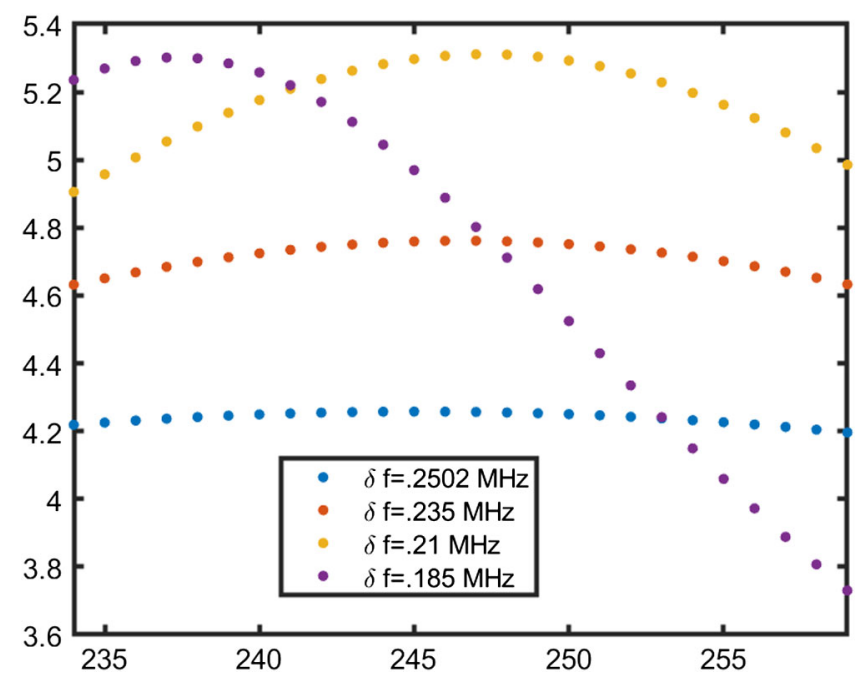

FIG. 19. Ratio of Touschek lifetime to that without HHC, with decreasing detuning. Distributed gaps with no guard bunches.

lifetime along the train, by a factor of 4.2. This occurs in spite of the substantial variation in the bunch form, suggesting that a flattopped distribution is not a primary requirement as far as lifetime is concerned.

With smaller detuning and consequent overstretching, we get a further increase in lifetime, but with increasing variation along the train. At the smallest detuning of Fig. 19, $\delta f=185 \mathrm{kHz}$, any advantage of overstretching is gone, since for half of the train the lifetime is smaller than for $\delta f=235 \mathrm{kHz}$.

\section{CONCLUSIONS AND OUTLOOK}

We have described an effective scheme to determine the equilibrium state of an arbitrary bunch train, subject to the 
wakefield from a passive harmonic cavity in its fundamental mode. The calculation proceeds by an iterative method with extremely robust convergence. The computation time is negligible, and the results agree with macroparticle simulations, which are much heavier calculations and also much more noisy.

The quick computation allows a convenient exploration of parameter space and, in particular, an examination of schemes to counter the bad effects of gaps in the bunch train. We have seen that, by distributing the empty buckets around the ring as much as possible, the bunch lengthening and centroid displacement can be made comparable to those of the complete fill. Although there is then considerable deviation from the flattopped distribution achieved in the complete fill, that turns out not to harm the Touschek lifetime. Also, most of the bunches can be given a flattopped form by invoking guard bunches adjacent to the gaps.

We have adopted a minimal physical model, with the only induced voltage (wakefield) coming from a single resonant mode of the harmonic cavity. With this, we could illustrate the power of a new technique in the simplest way. The next step toward a realistic model should be to include the induced voltage from the main accelerating cavity (beam loading). Since our formalism allows any number of resonators, this is a straightforward extension. In fact, we have revised the code to include the main cavity and have found that the iterative solution works as well as before, with only a factor of 2 increase in computer time.

Another refinement that could be significant is to include the effect of the usual short range wakefields from vacuum chamber corrugations. The magnitude of the effect on bunch lengthening can probably be judged by invoking a broadband resonator model of the machine impedance, which is normally applied with a $Q$ of the order of 1 . We have shown how to accommodate a low $Q$ in our formalism, by retaining exponential factors that could have been set to 1 in the present high- $Q$ calculations. We have not presented the full equations for low $Q$, but those follow after replacing (19) by the well-known formula for a broadband resonator [41] and proceeding with nearly the same steps as before. Our iterative determination of the diagonal term $v^{d}$ of Sec. VI might have to be revised.

One could also include higher-order modes of cavities and whispering gallery modes describing coherent synchrotron radiation [42].

Besides improving the physical model of the equilibrium state, an urgent matter is to study the stability of the equilibrium. This can, of course, be done by macroparticle simulations, but we would like to appeal as much as possible to the direct solution of the Vlasov-FokkerPlanck equation by the method of local characteristics, which proceeds with very low numerical noise [43]. The present study suggests possible reduced models of trains with gaps, in which the identity of some of the bunches would be enforced in one way or another. Our technique of exploiting geometric sums can help to simplify expressions for the induced voltage.

A special point of interest is the effect of overstretching on thresholds of instability. We have seen, without accounting for stability, that overstretching can give an additional increase in the Touschek lifetime.

\section{ACKNOWLEDGMENTS}

We thank several colleagues for advice and references to the literature: Karl Bane, Gabriele Bassi, Teresia Olsson, Boaz Nash, and Michael Borland. For many years, R. Warnock has been supported by an affiliation with Lawrence Berkeley National Laboratory as Guest Senior Scientist. Our work was supported in part by the U.S. Department of Energy, Contracts No. DE-AC0376 SF00515 and No. DE-AC02-05CH11231.

\section{APPENDIX A: SECOND EQUATION OF MOTION}

To derive the second equation of motion, note that the azimuthal location of a particle with revolution frequency $\omega_{0}+\Delta \omega$ is

$$
\theta=\left(\omega_{0}+\Delta \omega\right) t, \quad \theta=2 \pi s / C .
$$

If the cavity is at $\theta=0$, the $n$th passage of the cavity occurs at time $t_{n}$ such that

$$
2 \pi n=\left(\omega_{0}+\Delta \omega\right) t_{n}
$$

At that time, the cavity phase is

$$
\begin{aligned}
\phi_{n} & =\omega_{1} t_{n}=\omega_{1} \frac{2 \pi n}{\omega_{0}+\Delta \omega} \\
& \approx 2 \pi n \frac{\omega_{1}}{\omega_{0}}\left(1-\frac{\Delta \omega}{\omega_{0}}\right)=2 \pi n h\left(1-\frac{\Delta \omega}{\omega_{0}}\right) .
\end{aligned}
$$

The term $2 \pi n h$ on the right-hand side can be dropped, since it does not affect the applied voltage $V_{1} \sin \phi_{n}$ nor the induced voltage $V_{r}\left[\left(\phi_{n}-\phi_{0}\right) / k_{1}-m_{j} \lambda_{1}\right]$. Indeed, under substitution of that term, the argument $\phi_{n} / k_{1}=\phi_{n} \lambda_{1} / 2 \pi$ takes on the value $n h \lambda_{1}=n C$. Since $V_{r}$ is periodic with period $C$, it is not changed by the presence of the term $2 \pi n h$ in $\phi_{n}$.

With the definition (8), we then have

$$
(\Delta \phi)_{n+1}-(\Delta \phi)_{n}=-2 \pi h \frac{\Delta \omega}{\omega_{0}} .
$$

For highly relativistic particles above transition, the momentum compaction factor $\alpha$ can be written as 


$$
\alpha=-\frac{E_{0}}{\omega_{0}} \frac{\Delta \omega}{\Delta E} .
$$

Hence, with $\delta=\left(E_{n}-E_{0}\right) / E_{0}$, we have

$$
\frac{1}{T_{0}}\left[(\Delta \phi)_{n+1}-(\Delta \phi)_{n}\right]=2 \pi \frac{h}{T_{0}} \alpha \delta=\frac{2 \pi}{\lambda_{1}} \frac{h \lambda_{1}}{T_{0}} \alpha \delta=k_{1} c \alpha \delta .
$$

Passing to the corresponding differential equation, we obtain (15).

\section{APPENDIX B: PERTURBED SYNCHRONOUS PHASE}

To determine the perturbed synchronous phase, we put $k_{1} z_{i}+\phi_{0}=\phi_{0 i}$ in (16). We then see that the synchronous phase $\phi_{0 i}$ for the $i$ th bunch is defined by

$$
\begin{aligned}
& e V_{1} \sin \phi_{0 i}+e V_{r}\left(\frac{\phi_{0 i}-\phi_{0}}{k_{1}}-m_{i} \lambda_{1}\right)=U_{0}, \\
& \cos \phi_{0 i}=-\left(1-\sin ^{2} \phi_{0 i}\right)^{1 / 2} .
\end{aligned}
$$

It is the phase at which the force is zero, at the center of the distorted potential well.

In (B1), we have a nonlinear equation to solve for $\phi_{0 i}$. If Eq. (B1) can be solved, one can work out the dynamics for a new variable $\tilde{z}_{i}$ defined by

$$
k_{1} \tilde{z}_{i}=\phi_{i}-\phi_{0 i}
$$

where $\phi_{i}$ is the dynamical phase of the applied voltage when the particle arrives. That is, the applied voltage takes the form $V_{1} \sin \left(k_{1} \tilde{z}_{i}+\phi_{0 i}\right)$, and $\tilde{z}_{i}$ is zero at the minimum of the distorted potential well.

The scheme now involves a two-part iteration. In an iterate of part 1, the synchronous phases $\phi_{0 i}$ are determined by solving (B1) with a given function $V_{r}$. In a succeeding iterate of part 2, those $\phi_{0 i}$ are used to calculate the charge densities and, thus, to form a new value of $V_{r}$, by the algorithm described in Sec. X.

We programmed this scheme and found that it converges at a moderate current but runs into difficulty near the design current, because at that current we are getting close to the situation in which (B1) does not have a unique solution, owing to the advent of a doubly peaked charge density. At a moderate current, the results agree quite precisely with those from the simpler scheme based on the currentindependent $\phi_{0}$ and the original variables $z_{i}$.

Since the simpler scheme works at any current up to the design current and even far beyond, we have applied it for all further work. It is not necessary to base the coordinate system on the synchronous phases, but they can be found a posteriori as the location of the minima of the distorted potential wells computed using the $z_{i}$ as coordinates.

\section{APPENDIX C: REDUCTION TO THE CASE WITH ALL BUCKETS FILLED}

Here, our task is to reduce our general formula for the induced voltage to its form when all $\mathrm{rf}$ buckets are filled. We shall find that the resulting expression agrees to a close approximation with a formula well known in the literature. Thus, the following slightly complicated calculation serves as a good check on the preceding work.

In the equilibrium state, the bunches will all have the same charge density $\rho(z)$. We adapt the methods of Secs. IV and V. The total charge density will be

$$
\rho_{\mathrm{tot}}(z)=\sum_{p=-\infty}^{\infty} \sum_{j=1}^{h} \rho\left[z+(j-1) \lambda_{1}+p C\right] .
$$

By translating the integration variable, we get the induced voltage as

$$
\begin{aligned}
& V_{r}(z) \\
& =-e N \int W\left(z-z^{\prime}\right) \sum_{p=-\infty}^{\infty} \sum_{j=1}^{h} \rho\left[z^{\prime}+(j-1) \lambda_{1}+p C\right] d z^{\prime} \\
& =-e N \int_{-\Sigma}^{\Sigma}\left[\sum_{j=1}^{h} \sum_{p=-\infty}^{\infty} W\left[z-z^{\prime}+(j-1) \lambda_{1}+p C\right]\right] \rho\left(z^{\prime}\right) d z^{\prime} .
\end{aligned}
$$

In the notation of (21), the sum over $p$ in this expression is

$$
\mathcal{W}\left[z-z^{\prime}+(j-1) \lambda_{1}\right]
$$

which can be calculated from the formula (30). To apply the formula, we first show that

$$
\chi\left[z-z^{\prime}+(j-1) \lambda_{1}\right]=z-z^{\prime}+(j-1) \lambda_{1},
$$

to an excellent approximation. Since $0 \leq j-1 \leq h-1$ and $|z|,\left|z^{\prime}\right|<\Sigma$, we have

$-\frac{1}{C}\left(2 \Sigma+C-\lambda_{1}\right)<\frac{1}{C}\left[z-z^{\prime}+(j-1) \lambda_{1}\right]<\frac{2 \Sigma}{C}$.

From this, we can evaluate the ceiling function that appears in the definition (31) of $\chi$. At the lower and upper bounds of its argument from (C5), we have

$$
\begin{gathered}
\left\lceil-1+\left(\lambda_{1}-2 \Sigma\right) / C\right\rceil=0, \quad \text { since } 2 \Sigma \ll \lambda_{1}, \\
\lceil 2 \Sigma / C\rceil=1 .
\end{gathered}
$$

The evaluation (C7) occurs only for $j=1$ and only for the part of the integration where $z^{\prime}-z>0$. Since there are several hundred terms of similar magnitude in the sum 
over $j$, this case may safely be ignored. Thus, only the evaluation (C6) occurs, which implies that (C4) is correct.

Using (C4) in (C3) and (30), we now have (C2) reduced to a single sum:

$$
\begin{aligned}
V_{r}(z)= & -\frac{e N \omega_{r} R_{s} \eta}{Q} \int d z^{\prime} \rho\left(z^{\prime}\right) \\
& \times \sum_{j=1}^{h} \exp \left(-\frac{k_{r}}{2 Q}\left[z-z^{\prime}+(j-1) \lambda_{1}\right]\right. \\
& \left.\cdot \cos \left\{k_{r}\left[z-z^{\prime}+(j-1) \lambda_{1}\right]+\psi\right\}\right) .
\end{aligned}
$$

Once again, we have a geometric sum. Writing the cosine in terms of exponentials, one readily shows that

$$
\begin{aligned}
V_{r}(z)= & -\frac{e N \omega_{r} R_{s} \eta}{Q} \int d z^{\prime} \rho\left(z^{\prime}\right) \exp \left(-\frac{k_{r}}{2 Q} \theta\right) \\
& \times[\cos \theta \operatorname{Re} \tilde{\zeta}+\sin \theta \operatorname{Im} \tilde{\zeta}], \\
\theta= & z-z^{\prime}+\psi, \\
\tilde{\zeta}\left(k_{r}\right)= & \frac{1-r^{h}}{1-r}, \quad r=\exp \left[-k_{r} \lambda_{1}(i+1 / 2 Q)\right] .
\end{aligned}
$$

Note that $r$ is the same as in (28).

In analogy to (28), we define real polar variables by

$$
\tilde{\zeta}=\tilde{\eta} e^{-i \tilde{\psi}}
$$

Writing

$\cos \theta=\cos \left(k_{r} z+\psi\right) \cos \left(k_{r} z^{\prime}\right)+\sin \left(k_{r} z+\psi\right) \sin \left(k_{r} z^{\prime}\right)$,

and the analogous expression for $\sin \theta$, and applying the definition (41), we see that

$$
\begin{aligned}
V_{r}(z)= & -\frac{2 \pi e N \omega_{r} R_{s} \eta \tilde{\eta}}{Q} \exp \left(-k_{r} z / 2 Q\right) \\
& \times\left[\cos \left(k_{r} z+\psi+\tilde{\psi}\right) \operatorname{Re} \hat{\rho}-\sin \left(k_{r} z+\psi+\tilde{\psi}\right) \operatorname{Im} \hat{\rho}\right] .
\end{aligned}
$$

Now note the following identities and definitions:

$$
\begin{aligned}
\eta \tilde{\eta} & =\left|\frac{1}{1-r^{h}}\right|\left|\frac{1-r^{h}}{1-r}\right|=\left|\frac{1}{1-r}\right|=: \kappa, \\
\psi+\tilde{\psi} & =-\arg \left(\frac{1}{1-r^{h}}\right)-\arg \left(\frac{1-r^{h}}{1-r}\right)=-\arg \left(\frac{1}{1-r}\right) \\
& =\arg (1-r)=: \varphi .
\end{aligned}
$$

To evaluate $1-r$, notice that (for a third-harmonic cavity)

$$
\begin{aligned}
k_{r} \lambda_{1} & =3 k_{1} \lambda_{1}+\Delta k \lambda_{1}=6 \pi+\Delta k \lambda_{1}, \\
\Delta k & =k_{r}-k_{3}, \quad k_{3}=3 k_{1}, \\
\sin \left(k_{r} \lambda_{1}\right) & \approx \Delta k \lambda_{1}, \quad \cos \left(k_{r} \lambda_{1}\right) \approx 1,
\end{aligned}
$$

and

$$
1-r \approx 1-\left(1-k_{r} \lambda_{1} / 2 Q\right)\left(1-i \Delta k \lambda_{1}\right) \approx k_{r} \lambda_{1} / 2 Q+i \Delta k \lambda_{1} .
$$

From this, it follows that

$$
\kappa=\left|\frac{1}{1-r}\right|=\frac{f_{1}}{\left[\left(\omega_{r}-\omega_{3}\right)^{2}+(\Gamma / 2)^{2}\right]^{1 / 2}}, \quad \frac{\Gamma}{2}=\frac{\omega_{r}}{2 Q},
$$

where $\omega=k c$ and $f_{1}=c / \lambda_{1}$ is the rf frequency. Thus, we have Lorentzian resonant behavior, with half-width $\Gamma / 2$. Also

$$
\varphi=\arg (1-r)=\tan ^{-1}\left[\frac{\omega_{r}-\omega_{3}}{\Gamma / 2}\right] .
$$

Since the average current is $I_{\text {avg }}=e N / T_{1}=e N f_{1}$, we may write (C13) as

$$
\begin{aligned}
V_{r}(z)= & -4 \pi I_{\mathrm{avg}} R_{s}\left|\hat{\rho}\left(k_{r}\right)\right| \frac{\Gamma / 2}{\left[\left(\omega_{r}-\omega_{3}\right)^{2}+(\Gamma / 2)^{2}\right]^{1 / 2}} \\
& \times \cos \left[k_{r} z+\varphi+\arg \left(\hat{\rho}\left(k_{r}\right)\right] .\right.
\end{aligned}
$$

This is to be compared with a formula that is often quoted in the literature; see, for instance, Eq. (A7) and its derivation in Ref. [27]. By (A2) and (A5) of that paper and our definition (41),

$$
\begin{aligned}
\cos \psi_{\omega_{3}} & \approx \cos \varphi=\frac{\Gamma / 2}{\left[\left(\omega_{r}-\omega_{3}\right)^{2}+(\Gamma / 2)^{2}\right]^{1 / 2}}, \\
\hat{\rho}\left(k_{r}\right) & \approx \frac{1}{2 \pi} \tilde{\rho}\left(\omega_{3}\right)^{*}=\frac{1}{2 \pi} F e^{-i \Phi} .
\end{aligned}
$$

Also, $k_{r} z \approx \omega_{3} \tau$ so that (C19) becomes (with neglect of terms higher order in $\Delta k$ )

$$
V_{r}(z)=-2 I_{\mathrm{avg}} R_{s} F \cos \varphi \cos \left(\omega_{3} \tau+\varphi-\Phi\right),
$$

in agreement with (A7) of Ref. [27].

[1] A. Piwinski, The Touschek effect in strong focusing storage rings, Deutsches Elektronen-Synchrotron Report No. DESY 98-179, 1998. 
[2] A. Hofmann and S. Myers, Beam dynamics in a double rf system, CERN Report No. ISR-TH-RF/80-26, Geneva, 1980.

[3] J. M. Byrd and M. Georgsson, Lifetime increase using passive harmonic cavities in synchrotron light sources, Phys. Rev. Accel. Beams 4, 030701 (2001).

[4] M. Georgsson, A. Andersson, and M. Eriksson, Landau cavities at MAX-II, Nucl. Instrum. Methods Phys. Res., Sect. A 416, 465 (1998).

[5] J. M. Byrd, S. De Santis, M. Georgsson, G. Stover, J. D. Fox, and D. Teytelman, Commissioning of a higher harmonic rf system for the Advanced Light Source, Nucl. Instrum. Methods Phys. Res., Sect. A 455, 271 (2000).

[6] M. Pedrozzi et al., SLS operational performance with third harmonic superconducting system, in Proceedings of the 11th workshop on rf superconductivity, Lübeck, Germany.

[7] M. Svandrlik et al., Performance of the 3rd harmonic superconducting cavity at ELLETRA, in Proceedings of the 11th workshop on rf superconductivity, Lübeck, Germany.

[8] W. Anders and P. Kuske, HOM damped NC passive harmonic cavities at BESSY, in Proceedings of the 2003 Particle Accelerator Conference, p. 1186.

[9] D. Alesini et al., The DAФNE 3rd harmonic cavity, in Proceedings of the 19th Particle Accelerator Conference, Chicago, IL, 2001 (IEEE, Piscataway, NJ, 2001), p. 885.

[10] N. Towne and J. Rose, Bunch lengthening harmonic system for NSLS-II, in Proceedings of the 11th European Particle Accelerator Conference, Genoa, 2008 (EPS-AG, Genoa, Italy, 2008), paper MOPP152.

[11] A. Blednykh et al., Harmonic cavity performance for NSLS-II, in Proceedings of the 21st Particle Accelerator Conference, Knoxville, TN, 2005 (IEEE, Piscataway, NJ, 2005), p. 2544.

[12] G. Bassi and J. Tagger, Longitudinal beam dynamics with a higher harmonic cavity for bunch lengthening, Int. J. Mod. Phys. A 34, 1942040 (2019).

[13] G. Skripka et al., Commissioning of the harmonic cavities in the MAX IV $3 \mathrm{GeV}$ storage ring, in Proceedings of IPAC 2016, Busan, Korea, paper WEPOW035.

[14] H. Feng et al., Design study on higher harmonic cavity for ALS-U, in Proceedings of IPAC 2019, Melbourne, Australia, WEPRB081.

[15] M. P. Kelly et al., Superconducting harmonic cavity for the Advanced Photon Source Upgrade, in Proceedings of IPAC 2015, Richmond, VA, paper WEPTY005.

[16] http://wiki-sirius.lnls.br/mediawiki/index.php/Machine:RF_ System\#Harmonic_Cavity.

[17] C. Schroer et al., PETRA IV: The ultralow-emittance source project at DESY, J. Synchrotron Radiat. 25, 1277 (2018).

[18] A. Streun, T. Garvey, L. Rivkin, V. Schlott, T. Schmidt, P. Willmott, and A. Wrulich, SLS-2-The upgrade of the Swiss Light Source, J. Synchrotron Radiat. 25, 631 (2018).

[19] N. Carmignani, J. Jacob, B. Nash, and S. White, Harmonic rf system for the ESRF EBS, in Proceedings of IPAC 2017, Copenhagen, Denmark, paper THPAB003.

[20] http://diamond.ac.uk/Home/About/Vision/Diamond-II.html.

[21] T. Olsson, I. P. S. Martin, and R. Bartolini, Harmonic cavity design choice for lifetime increase in Diamond-II, in Proc. IPAC 2019, Melbourne, Australia, paper
TUPGW078, http://diamond.ac.uk/Home/About/Vision/ Diamond-II.html.

[22] C. Steier et al., Status of the conceptual design of ALS-U, in Proceedings of IPAC 2017, paper TUOCS3.

[23] R. Warnock and K. Bane, Numerical solution of the Haïssinski equation for the equilibrium state of a stored electron beam, Phys. Rev. Accel. Beams 21, 124401 (2018).

[24] T. Olsson, T. J. Cullinan, and $\AA$. Andersson, Self-consistent calculation of transient beam loading in electron storage rings with passive harmonic cavity, Phys. Rev. Accel. Beams 21, 120701 (2018).

[25] S. Krinsky and J. M. Wang, Longitudinal instabilities of bunched beams subject to a non-harmonic rf potential, Part. Accel. 17, 109 (1985).

[26] R. A. Bosch, Instabilities driven by higher-order modes in a radio frequency system with a passive higher-harmonic cavity, Phys. Rev. Accel. Beams 8, 084401 (2005).

[27] M. Venturini, Passive higher-harmonic rf cavities with general settings and multibunch instabilities in electron storage rings, Phys. Rev. Accel. Beams 21, 114404 (2018).

[28] M. Venturini, Harmonic cavities and the transverse mode coupling instability driven by a resistive wall, Phys. Rev. Accel. Beams 21, 024402 (2018).

[29] R. R. Lindberg, Theory of longitudinal coupled-bunch instabilities in a storage ring for arbitrary rf potentials, Phys. Rev. Accel. Beams 21, 124402 (2018).

[30] N. Towne, Longitudinal simulations and measurements of stretched bunches, Phys. Rev. Accel. Beams 4, 114401 (2001).

[31] J. M. Byrd, S. De Santis, J. Jacob, and V. Serriere, Transient beam loading effects in harmonic rf systems for light sources, Phys. Rev. Accel. Beams 5, 092001 (2002).

[32] P. F. Tavares, Å. Andersson, A. Hansson, and J. Breunlin, Equilibrium bunch density distribution with passive harmonic cavities in a storage ring, Phys. Rev. Accel. Beams 17, 064401 (2014).

[33] N. Yamamoto, T. Takahashi, and S. Sakanaka, Reduction and compensation of the transient beam loading effect in a double rf system of synchrotron light sources, Phys. Rev. Accel. Beams 21, 012001 (2018).

[34] G. Wüustefeld, A. Jankowiak, J. Knobloch, and M. Ries, Simultaneoous long and short electron bunches in the BESSY-II storage ring, Proc. IPAC 2011, San Sebastian, Spain, paper THPC014.

[35] S.-K. Tian, Y. Jiao, and J.-Q. Wang, Bunch length manipulation in a diraction-limited storage ring, Chin. Phys. C 39, 127001 (2015).

[36] E. L. Allgower and K. Georg, Introduction to Numerical Continuation Methods (SIAM, Philadelphia, 2003).

[37] Z. Pan, S. De Santis, T. Hellert, C. Steier, C. Sun, C. Tang, and M. Venturini, Beam loading transients and bunch shape in the operation of passive harmonic cavities in the ALS-U, in Proceedings of IPAC 2018, Vancouver, BC, Canada, 2018, paper THPAK037.

[38] M. Borland (private communication).

[39] User's manual for ELEGANT, http://ops.aps.anl.gov/ manuals/elegant_latest/elegant.html.

[40] N. Carmignani et al., Lifetime improvements with a harmonic rf system for the ESRF EBS, in Proceedings of IPAC 2016, Busan, Korea, paper TUPOR001. 
[41] A. W. Chao, Physics of Collective Beam Instabilities in High Energy Accelerators (Wiley, New York, 1993), Eq. (2.84).

[42] R. Warnock, J. Bergstrom, and M. Klein, Coherent synchrotron radiation in whispering gallery modes: Theory and evidence, ICFA Beam Dynamics Newsletter 63, 32 (2013), http://www-bd.fnal.gov/icfabd/Newsletter63.pdf.

[43] R. Warnock, Study of bunch instabilities by the nonlinear Vlasov-Fokker-Planck equation, Nucl. Instrum. Methods Phys. Res., Sect. A 561, 186 (2006). 